\title{
Observational Signatures of Transverse Magnetohydrodynamic Waves and Associated Dynamic Instabilities in Coronal Flux Tubes
}

\author{
P. Antolin ${ }^{1}$, I. De Moortel ${ }^{1}$, T. Van Doorsselaere ${ }^{2}$, and T. Yokoyama ${ }^{3}$ \\ ${ }^{1}$ School of Mathematics and Statistics, University of St. Andrews, St. Andrews, Fife KY16 9SS, UK; patrick.antolin@st-andrews.ac.uk \\ ${ }^{2}$ Centre for mathematical Plasma Astrophysics, Mathematics Department, KU Leuven, Celestijnenlaan 200B bus 2400, B-3001 Leuven, Belgium \\ ${ }^{3}$ Department of Earth and Planetary Science, The University of Tokyo, Hongo, Bunkyo-ku, Tokyo 113-0033, Japan \\ Received 2016 November 23; revised 2017 January 27; accepted 2017 February 2; published 2017 February 22
}

\begin{abstract}
Magnetohydrodynamic (MHD) waves permeate the solar atmosphere and constitute potential coronal heating agents. Yet, the waves detected so far may be but a small subset of the true existing wave power. Detection is limited by instrumental constraints but also by wave processes that localize the wave power in undetectable spatial scales. In this study, we conduct 3D MHD simulations and forward modeling of standing transverse MHD waves in coronal loops with uniform and non-uniform temperature variation in the perpendicular cross-section. The observed signatures are largely dominated by the combination of the Kelvin-Helmholtz instability (KHI), resonant absorption, and phase mixing. In the presence of a cross-loop temperature gradient, we find that emission lines sensitive to the loop core catch different signatures compared to those that are more sensitive to the loop boundary and the surrounding corona, leading to an out-of-phase intensity and Doppler velocity modulation produced by KHI mixing. In all of the considered models, common signatures include an intensity and loop width modulation at half the kink period, a fine strand-like structure, a characteristic arrow-shaped structure in the Doppler maps, and overall line broadening in time but particularly at the loop edges. For our model, most of these features can be captured with a spatial resolution of 0!' 33 and a spectral resolution of $25 \mathrm{~km} \mathrm{~s}^{-1}$, although we do obtain severe over-estimation of the line width. Resonant absorption leads to a significant decrease of the observed kinetic energy from Doppler motions over time, which is not recovered by a corresponding increase in the line width from phase mixing and KHI motions. We estimate this hidden wave energy to be a factor of 5-10 of the observed value.
\end{abstract}

Key words: magnetohydrodynamics (MHD) - Sun: activity - Sun: corona - Sun: filaments, prominences Sun: oscillations

Supporting material: animation

\section{Introduction}

Research in the last decade has shown that transverse magnetohydrodynamic (MHD) waves permeate the solar atmosphere (Aschwanden et al. 1999; Nakariakov et al. 1999; Tomczyk et al. 2007; Lin 2011; Arregui et al. 2012; De Moortel \& Nakariakov 2012; Anfinogentov et al. 2015). These MHD waves are of particular interest due to their ability to carry large amounts of energy, and thus potentially to contribute significantly to coronal heating (Uchida \& Kaburaki 1974; Wentzel 1974; McIntosh et al. 2011). A peculiar characteristic of these waves, particularly those with high amplitudes, has been their very fast damping. The leading theory explaining this behavior is resonant absorption for the case of standing modes (Goossens et al. 2002, 2006; Van Doorsselaere et al. 2004; Goossens et al. 2011), and mode coupling for the case of propagating modes (Allan \& Wright 2000; Pascoe et al. 2010; Terradas et al. 2010; Verth et al. 2010; De Moortel et al. 2016).

Resonant absorption (and mode coupling), is an ideal process of energy transfer between different wave modes (Ionson 1978; Hollweg 1987; Hollweg \& Yang 1988; Sakurai et al. 1991), and has been shown to be very efficient and robust (Terradas et al. 2008b; De Moortel \& Nakariakov 2012; Pascoe et al. 2011). Resonant absorption predicts that in the presence of density inhomogeneity, the global kink mode can resonantly couple with azimuthal Alfvén waves. In the classical picture of coronal loops with a density gradient between the inside and the outside of the flux tube, the resonance layer is expected to exist at the boundaries (edges) of loops. The global kink mode, which consists of a purely transverse displacement of the loop, then behaves as local azimuthal Alfvén waves for most of the oscillation time. This means that most of the displacement (and thus the energy) from such waves is azimuthal and localized in the loop shell, rather than being transverse and global.

Quantifying the amount of wave power in the solar atmosphere is essential for determining the role of waves in coronal heating. Based on the above information, the ideal instruments to detect most of the wave power in Alfvénic modes are spectroscopic instruments. However, even when using such instruments, line-of-sight (LOS) effects are expected to be important since Doppler velocities (and therefore wave power) are significantly reduced due to the loss of spatial velocity coherency from phase mixing and the LOS integration across multiple waveguides (De Moortel \& Pascoe 2012). In theory, the remaining wave power should still be detectable, albeit with sufficiently high resolution, in the line broadening as well as in the distribution of power in the frequency spectrum. McIntosh \& De Pontieu (2012) analyzed Coronal Multi-channel Polarimeter (CoMP) spectroscopic measurements of the global corona, where clear periodic Doppler shifts are observed (Tomczyk et al. 2007; Van Doorsselaere et al. 2008a). The authors showed that the nonthermal line widths are correlated with the Doppler components, strongly suggesting an important wave contribution in the line widths. Supporting this conclusion, also using CoMP data, Morton et al. (2016) have shown that there is a power 
spectrum enhancement at $3 \mathrm{mHz}$ everywhere in the corona, suggesting a p-mode origin for the kink waves (Khomenko \& Collados 2015; Cally et al. 2016).

The wave energy content hidden in the line broadening of coronal lines is still unclear. Through Monte Carlo simulations and comparison with the CoMP observations, McIntosh \& De Pontieu (2012) estimate the wave amplitudes to be between 25 and $56 \mathrm{~km} \mathrm{~s}^{-1}$. Other observational reports place the nonthermal component peak at about $15-25 \mathrm{~km} \mathrm{~s}^{-1}$ (with a long tail up to $40 \mathrm{~km} \mathrm{~s}^{-1}$ or so) in coronal lines (Hara \& Ichimoto 1999; Doschek et al. 2007; Hara et al. 2008), and with a tendency toward larger values for the LOS transverse to the loop plane, supporting an Alfvénic origin. Observations at higher resolution in transition region lines (De Pontieu et al. 2015) or prominence observations in chromospheric lines show similar values, suggesting that non-thermal velocities and spatial resolution are uncorrelated to some extent (Parenti \& Vial 2007; Parenti et al. 2015). Such results can be interpreted as evidence for Alfvénic turbulence, a scenario also supported by numerical modeling (e.g., AsgariTarghi et al. 2014; Cranmer \& Woolsey 2015; Woolsey \& Cranmer 2015). This scenario is further supported by power spectrum analysis of Doppler shift oscillations from CoMP, performed by De Moortel et al. (2014), in which a shift of the power is observed toward high frequencies at the loop apex and is interpreted as a possible signature of the expected turbulence cascade.

Besides the determination of the wave energy, the second biggest obstacle for Alfvénic wave heating of the corona is determining an efficient dissipation mechanism. The generation of turbulence from waves is considered as a possible solution to this problem. Spatial inhomogeneities, wave-to-wave interaction, and, particularly, instabilities, can be major sources of turbulence. Alfvénic waves such as kink waves can become unstable due to shear motions (Kelvin-Helmholtz instability, $\mathrm{KHI}$ ) at the boundaries (shell) of flux tubes (Terradas et al. 2008a). This instability has been modeled in photospheric, chromospheric, and coronal conditions (Karpen et al. 1993; Ofman et al. 1994; Poedts et al. 1997; Ziegler \& Ulmschneider 1997). In coronal loops, this instability can be obtained even for small-amplitude $\left(\lesssim 3 \mathrm{~km} \mathrm{~s}^{-1}\right.$ ) standing kink modes (Antolin et al. 2014). The ease with which the KHI can set in was recently confirmed by Zaqarashvili et al. (2015), who consider straight, twisted, and rotating magnetic structures. For the latter, the twist provides an inhibiting effect on the instability, which, however, can occur when the kinetic energy of the rotation is more than the magnetic energy of the twist. This was recently confirmed numerically by Murawski et al. (2016).

In the formation of turbulence from KHI associated with transverse MHD waves, a myriad of vortices and current sheets are formed along the flux tube which may contribute to the heating through viscous and resistive dissipation, but also through (component) magnetic reconnection, as suggested in Antolin et al. (2014). We refer to such KHI vortices as Transverse Wave Induced Kelvin-Helmholtz (TWIKH) vortices, or TWIKH rolls. Through mixing and heating, combined with LOS effects, TWIKH rolls lead to strand-like structures in intensity images in coronal loops (Antolin et al. 2014, 2016) and thread-like structures in prominences (Antolin et al. 2015). The KHI associated with transverse MHD waves thus cannot only have important consequences for the thermodynamic evolution of loops but must also have important consequences for their morphology. Not only does this model show that strands are not a unique feature of reconnection (Klimchuk 2006), but also that such structures cannot be independent. Indeed, as was recently shown by Magyar \& Van Doorsselaere (2016), an initially multi-stranded loop would rapidly lose such a state due to a transverse MHD wave and, more precisely, due to the KHI and phase mixing that is produced.

The KHI combined with resonant absorption and phase mixing in a coronal loop has important observational consequences that can explain several commonly detected features in observations. In Antolin et al. (2015), we characterized a set of imaging and spectroscopic observables for such waves traveling in prominences, providing an explanation for the observed phase difference between the LOS velocity signal and the transverse (in the plane-of-the-sky (POS) motion) observed by Hinode/SOT and IRIS (Okamoto et al. 2015), and potentially the thread-like structure that is usually seen accompanying the transverse motions of prominence threads (Okamoto et al. 2016). Other predicted observables from the combined KHI and resonant absorption mechanisms were the thinning and fading in cool lines, which are accompanied by broadening and intensity enhancement in hot lines, providing further explanation for the observed prominence threads disappearing in the $\mathrm{Ca}$ II $\mathrm{H}$ line at roughly $10^{4} \mathrm{~K}$ followed by their appearance in the hotter Si IV line at $10^{5} \mathrm{~K}$.

Recently, in Antolin et al. (2016), we demonstrated that for imaging instruments with spatial resolution such as that of SDO/AIA, TWIKH rolls can lead to an apparently decay-less oscillation for low-amplitude kink modes, due to the combined effect of periodic brightening from the vortices and their coherent motion. This effect, combined with the intensity dimming or enhancement produced from the KHI mixing, provides a possible physical explanation for observed decayless oscillations with AIA (Nisticò et al. 2013; Anfinogentov et al. 2013), a phenomenon that seems to be ubiquitous in active regions (Anfinogentov et al. 2015). Furthermore, we have shown that when a non-uniform temperature variation exists across the loop, different emission lines that are sensitive to different parts of the loop can catch the effect from the TWIKH rolls to varying degrees, leading to a temperaturedependent (and thereby density-dependent) period from the effects of phase mixing. Nakariakov et al. (2016) also proposed another explanation for the decay-less effect, framed in terms of self-oscillations at the natural loop frequency produced by quasi-steady flows.

In this paper, we examine the observable quantities from numerical simulations of standing transverse MHD waves in coronal loops, including both linear and non-linear effects such as the KHI, with forward modeling targeting both imaging and spectroscopic instruments. In particular, we focus on determining the instrumental requirements to detect the mechanisms at play for comparison with future coronal observations. This paper is organized as follows. In Section 2, we explain the numerical setup and the forward modeling. In Section 3, we analyze the results from the numerical model, which are then forward modeled and analyzed in Section 4. In Section 5, further forward modeling is performed targeting the spatial and spectral resolution of current instruments to test the resilience of our results during observations. We discuss our findings and conclude in Section 6. 


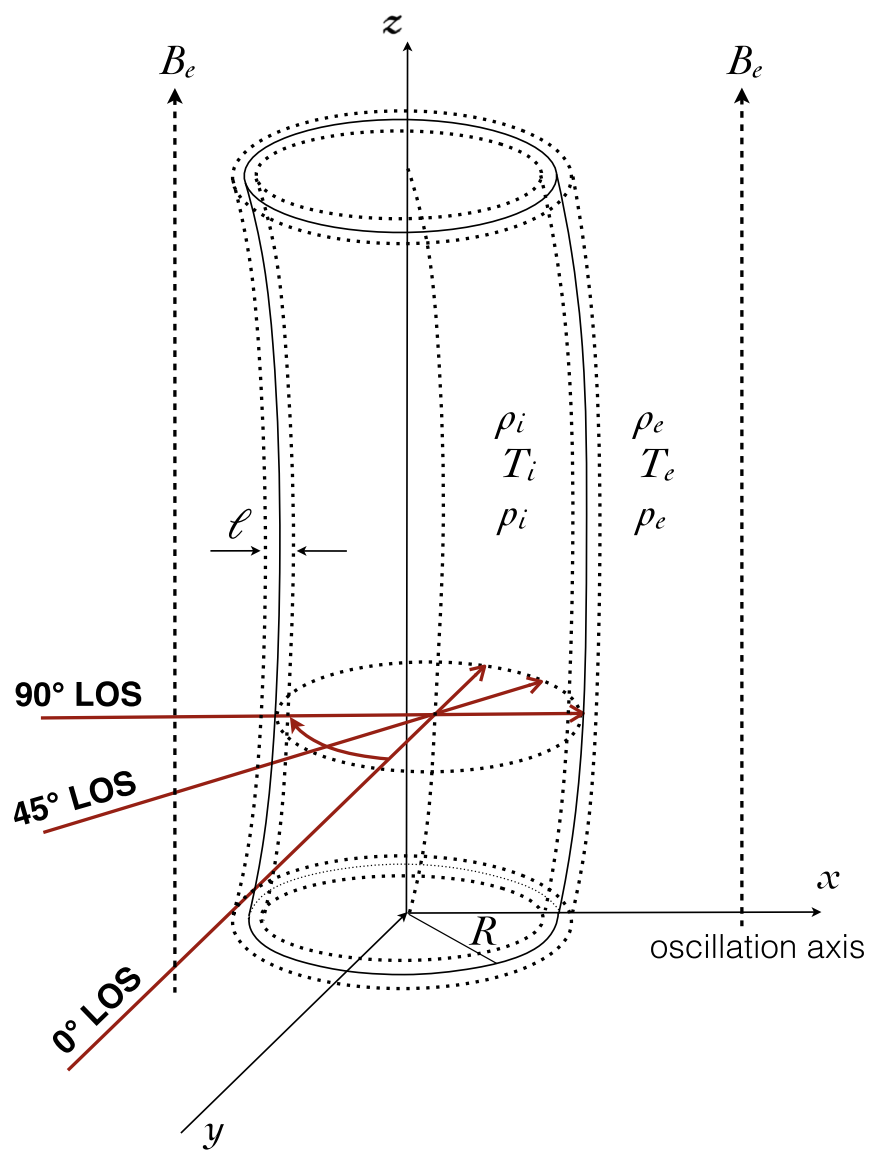

Figure 1. Sketch of our loop with radius $R$ undergoing a fundamental kink mode perturbation along $x$. The quantities $\rho, T, p$, and $B$ denote the density, temperature, pressure, and longitudinal magnetic field, respectively. The subindexes $i$ and $e$ denote the internal (within the loop) and external values (ambient corona). The LOS angles $0^{\circ}, 45^{\circ}, 90^{\circ}$ for the forward modeling are shown as red directional rays.

\section{Numerical Model}

Coronal loops are observed to be very dynamic, continuously appearing and disappearing across temperature passbands in imaging instruments such as AIA. Due to the longer cooling timescales with respect to the heating timescales, it is expected and observed that loops are usually in a state of cooling (Viall $\&$ Klimchuk 2012). Due to their higher densities with respect to the ambient corona (which is the main reason why we actually observe them as distinctive features in AIA passbands), the plasma inside will cool faster than the ambient plasma, a scenario which leads to different temperatures compared to the surroundings. For this reason, in this work we have considered two different initial atmospheres for our loop: one presenting a density and temperature contrast with the ambient corona but with a uniform magnetic field throughout, and another with a density and magnetic field contrast with the ambient corona but with uniform temperature throughout. In both cases, we initially have hydrostatic pressure balance.

\subsection{Initial Setup}

In model 1, our 3D MHD numerical model is the same as that in Antolin et al. (2014) where we take a loop with a density and temperature contrast with respect to the ambient low- $\beta$ coronal atmosphere. The loop is initially in hydrostatic equilibrium and has density and temperature ratios of $\rho_{i} / \rho_{e}=3$ and $T_{i} / T_{e}=1 / 3$, respectively, where the index $i$ (e) denotes internal (external) values. The magnetic field is uniformly set to $B_{0}=22.8 \mathrm{G}$. We initially take $T_{i}=10^{6} \mathrm{~K}$ and $\rho_{i}=3 \times 10^{9} \mu m_{p} \mathrm{~g} \mathrm{~cm}^{-3}\left(\mu=0.5\right.$ and $m_{p}$ is the proton mass), and the density profile across the loop is set as follows (shown in Figure 2):

$$
\rho(x, y)=\rho_{e}+\left(\rho_{i}-\rho_{e}\right) \zeta(x, y),
$$

where

$$
\zeta(x, y)=\frac{1}{2}(1-\tanh (b(r(x, y)-1))) .
$$

In these equations, $x$ and $y$ denote the coordinates in the plane perpendicular to the loop axis, and $z$ is along its axis. The $r(x, y)=\sqrt{x^{2}+y^{2}} / R$ term denotes the normalized radius of the loop at position $(x, y)$ and $b$ sets the width of the boundary layer (the loop shell). In our model, we set $b=8$ leading to $\ell / R \approx 0.4$, where $\ell$ denotes the width of the boundary layer and $R$ is the radius of the loop (see Figure 1). The length $L$ of the loop is $200 R$, and we set $R=1 \mathrm{Mm}$.

In the second numerical setup, model 2 , the temperature is uniformly set throughout to $T=7 \times 10^{5} \mathrm{~K}$. The density contrast is the same as in the first model. The magnetic field varies with the inverse density profile in order to maintain hydrostatic pressure balance (with a minimal variation from $B_{e}=11 \mathrm{G}$ to $\left.B_{i}=10.8 \mathrm{G}\right)$.

The loop in both models is subject to a perturbation mimicking a fundamental kink mode (longitudinal wavenumber $k R=$ $\pi R / L \approx 0.015)$ by initially imposing a perturbation along the loop for the transverse $x$-velocity component, according to $v_{x}(x, y, z)=v_{0} \cos (\pi z / L) \zeta(x, y)$, where $v_{0}$ is the initial amplitude. The corresponding kink phase speeds are $c_{k}=$ $\sqrt{\left(\rho_{i} v_{A_{i}}^{2}+\rho_{e} v_{A_{e}}^{2}\right) /\left(\rho_{i}+\rho_{e}\right)} \approx 1574 \mathrm{~km} \mathrm{~s}^{-1}$ and $752 \mathrm{~km} \mathrm{~s}^{-1}$, respectively, for models 1 and 2, where $v_{A_{i}}$ and $v_{A_{e}}$ denote the internal and external Alfvén speeds, which equal 1285 and $2225 \mathrm{~km} \mathrm{~s}^{-1}$, respectively, in model 1 and 608 and $1075 \mathrm{~km} \mathrm{~s}^{-1}$ in model 2. Here we present results for $v_{0}=0.05 c_{s}$, with $c_{s}$ being the external sound speed in each model. This corresponds to $v_{0}=15$ and $7 \mathrm{~km} \mathrm{~s}^{-1}$ in models 1 and 2 , respectively.

\subsection{Numerical Setup}

We perform the 3D MHD simulation described above with the CIP-MOCCT scheme (Kudoh et al. 1999). The MHD equations are solved while excluding gravity and loop curvature, which are second-order factors for the present work. Furthermore, the effects of radiative cooling and thermal conduction are also neglected, as they are expected to be unimportant due to their longer timescales compared to that set by the kink wave (see also Cargill et al. 2016).

The numerical box is $512 \times 256 \times 50$ points in the $x, y$, and $z$ directions, respectively. Thanks to the symmetric properties of the kink mode, only half of the plane in $y$ and half of the loop are modeled (from $z=0$ to $z=100 \mathrm{R}$ ), and we set symmetric boundary conditions in all of the boundary planes, except for $x$ where periodic boundary conditions are imposed. In order to minimize the influence from side boundary conditions (along $x$ and $y$ ), the spatial grids in $x$ and $y$ are non-uniform with exponentially increasing values for distances beyond the maximum displacement. The maximum distance from the center in $x$ and $y$ is $\approx 16 R$. The spatial 

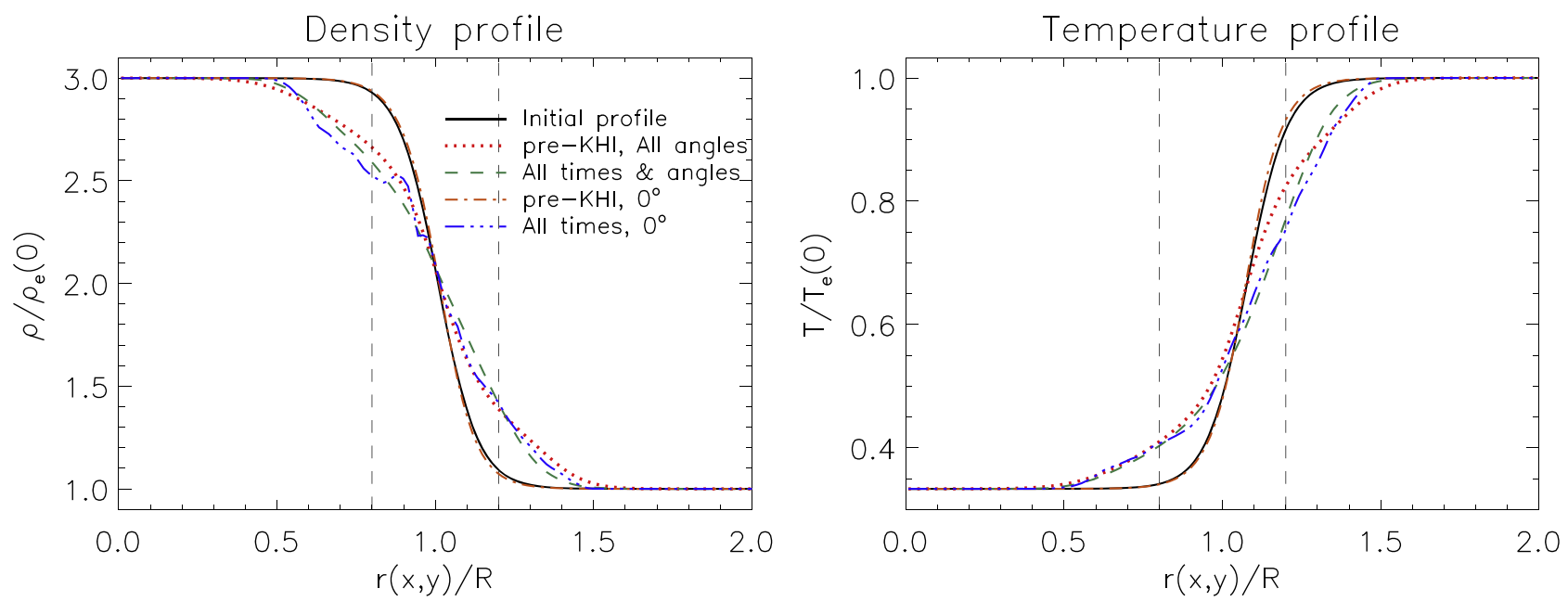

Figure 2. Initial density (left) and temperature (right) profiles (solid curves) across a perpendicular cross-section of the loop corresponding to model 1 . The boundary layer width $\ell$ is roughly $0.4 R$. Overlaid are their respective averages over all angles and times prior to KHI onset (dotted curves), the averages over all times and angles (dashed curves), the averages over times prior to KHI along the $y$-direction crossing the loop center $\left(0^{\circ}\right)$ in the Lagrangian frame of reference (dotted-dashed curves), and the averages over the same direction but over all times (dotted-dotted-dashed curves). The vertical dashed lines roughly denote the start and end of the initial boundary layer.

resolution at the loop's location is $0.0156 R=15.6 \mathrm{~km}$. The code includes explicit resistive and viscous coefficients which are, however, set to very low values in the current simulation. The simulation can therefore be considered ideal to a certain extent. However, from a parameter study, we estimate that the effective Reynolds and Lundquist numbers in the code are of the order of $10^{4}-10^{5}$. We have checked that the energy is conserved to high accuracy in the whole numerical box in the current simulation.

\section{Results-General Characteristics}

\subsection{Two Mechanisms Combined: Resonant Absorption and the KHI}

Following the initial perturbation with amplitudes of $v_{0}=15 \mathrm{~km} \mathrm{~s}^{-1}$ and $v_{0}=7 \mathrm{~km} \mathrm{~s}^{-1}$ for models 1 and 2 , respectively, the loop starts oscillating with periods of $P=255 \mathrm{~s}$ and $P=530 \mathrm{~s}$, respectively, closely corresponding to the period of the fundamental mode $2 L / c_{k}$. The maximum displacement of the loop in each model is 440 and $430 \mathrm{~km}$, that is, $0.44 R$ and $0.43 R$, respectively. As expected, resonant absorption rapidly sets in at the loop boundary. Energy is then transferred from global transverse oscillations to local azimuthal oscillations within the loop boundary, which is clearly visible in the $z$-component of the vorticity in the animated Figure 4.

In addition to resonant absorption, a second mechanism sets in. As mentioned in the Introduction, the kink mode produces a velocity shear at the boundary of the flux tube between the dipole-like external flow and the purely transverse motion of the kink mode. Both motions are part of the kink mode, with the former (dipole-like flow) being produced by the quadrupolar terms in the wave equation (Goossens et al. 2014; Yuan \& Van Doorsselaere 2016). In the presence of a density gradient, the shear is amplified by the presence of the (azimuthal) resonant flow. This shear generates the KHI, as recently demonstrated analytically by Zaqarashvili et al. (2015). In Figure 3, which shows snapshots of the emissivity of a cross-section of half the loop at the apex, we can see the formation of the initial vortices for both models (see also the animated Figure 4). Three vortices appear first, and half a period later four vortices appear, indicating that $m=3$ and 4 are the most unstable modes, which agrees with theoretical results (see Equation (58) in Zaqarashvili et al. 2015).

The KHI broadens the initial boundary layer, the extent of which can be seen in Figure 2. In this figure, we plot the density and temperature profiles averaged over time and angles around the cylindrical cross-section at the center of the loop for times prior to $\mathrm{KHI}$ onset and also over the entire simulation. Prior to the onset of KHI, the flux tube suffers a periodic compression and rarefaction at the loop boundary, leading to a deformation of the loop's cross-section. The compression and rarefaction occur, respectively, at the head and tail of the flux tube (along the axis of oscillation). This squashing effect is produced by the combination of fluting modes and the inertia produced by the wave (which acts differently on the loop's core and shell), and is completely reversible (the boundary shape in the $x$-direction goes back to the original shape each time the flux tube passes through the initial position). On the other hand, the KHI has an irreversible smoothing effect over all angles and is maximal at the sides of the flux tube that are perpendicular to the direction of oscillation. This is expected since these are the locations of maximum velocity shear. At the end of the simulation, the center of the loop is roughly back to the initial position and the loop core is squashed in the oscillation direction. The KHI therefore leads to elliptically shaped loop cores with boundary layers that have roughly linear density gradients and with the major axis along the direction of oscillation, as seen in Figure 4.

\subsection{Compressive Vortices and the Strand-like Structure in EUV Emission Lines}

The KHI produces vortices (TWIKH rolls) in the transverse cross-section along most of the flux tube's length. Large-scale vortices are generated first (at the location of the resonance), which rapidly break up into smaller and smaller vortices. As shown in Figure 4, such vortices mix the external ambient plasma with the internal loop plasma and produce current 

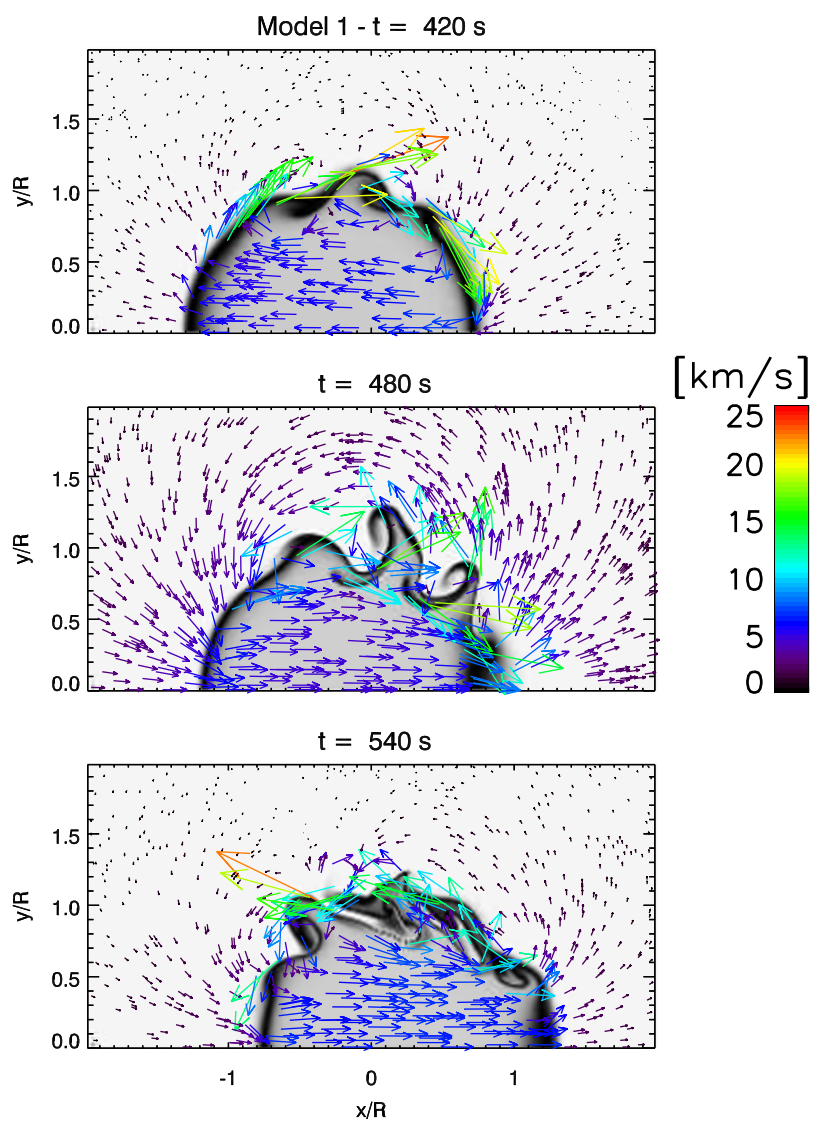
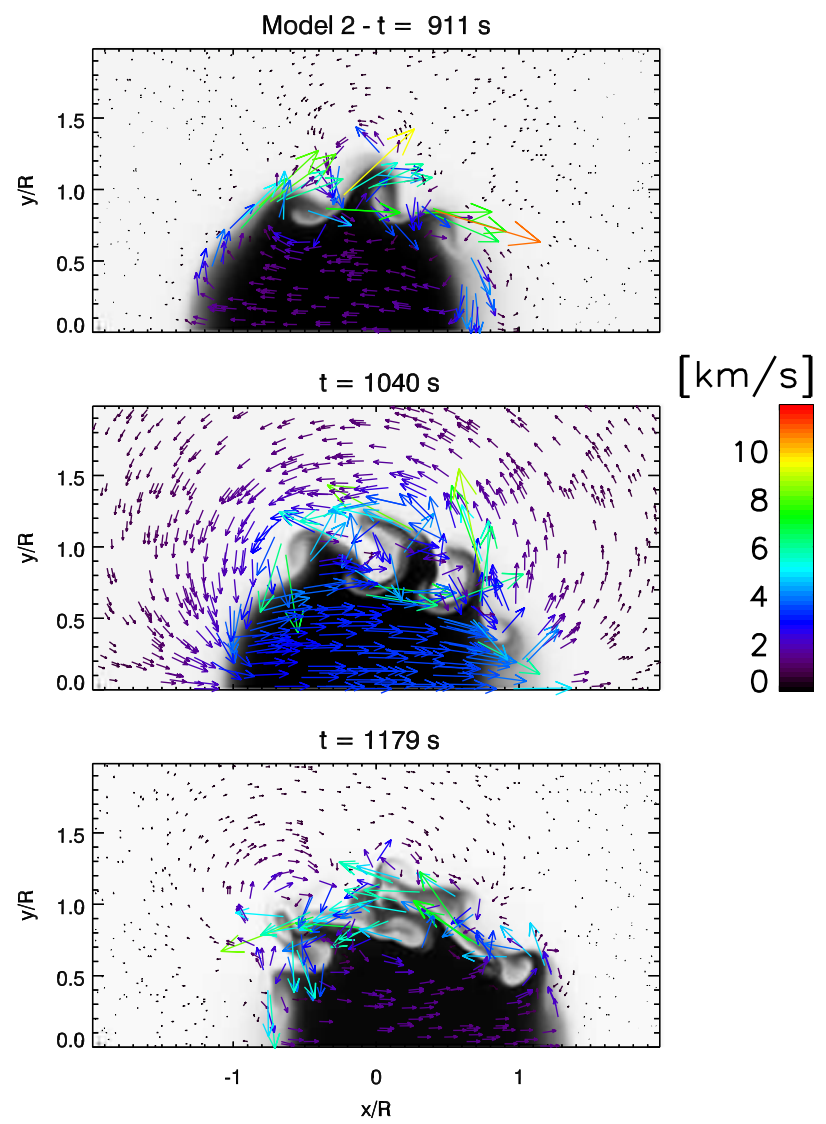

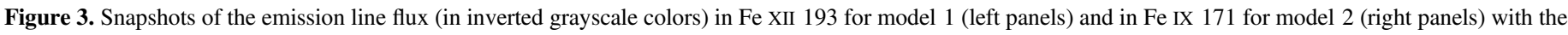

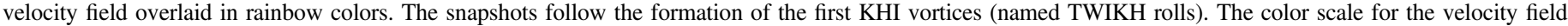
incorporates the extrema over the entire simulation.

sheets as well as locations of viscous and resistive dissipation. Comparing the results from model 1 and model 2 suggests that most of the change in loop temperature produced in the present scenario seems to come from mixing with the external plasma and not from actual wave dissipation. As shown by Figure 4 (and the online animated version), the region corresponding to positive temperature change in model 2 is the inner shell region where the density is reduced due to the KHI, while the outer shell region becomes denser and colder. The changes of temperature are thus mainly due to a change in density, and are thus mostly adiabatic rather than linked to wave dissipation (Karampelas et al. 2017).

The vortices (or roll-ups in 3D) have a fast MHD mode nature, in that they are able to compress the plasma and therefore significantly deform the density and temperature structure in the transverse plane of the flux tube, similar to the vortices generated through Alfvénic vortex shedding (Gruszecki et al. 2010). This is especially true in chromospheric conditions (Antolin et al. 2015) or for mild amplitude perturbations above $0.01 v_{A_{i}}$. Such vortices are therefore regions of enhanced emissivity. This can be clearly seen in Figure 3 where the TWIKH rolls have higher intensity (dark in the color inverted image). Due to the optically thin nature of the corona, the emission from these vortices adds up along the LOS, resulting in clear, strand-like structure in EUV intensity images of the loop, as first shown in Antolin et al. (2014, 2015).

\section{Forward Modeling the Uniform and Non-uniform Temperature Models}

\subsection{Emission Lines, LOS and Instrumental Setup}

To obtain observable quantities, the results from the numerical simulations are forward modeled using the FoMo code (Van Doorsselaere et al. 2016), which calculates the optically thin emission of coronal lines based on the CHIANTI atomic database (Dere et al. 2009).

We choose the Fe IX 171 and Fe XII 193 emission lines, which have rest wavelengths at 171.073 and $193.509 \AA$, and maximum formation temperatures of $\log T=5.93$ and $\log T=6.19$, respectively. For model 1 , the Fe IX 171 line is more tuned to detect the plasma response at the core of the loop, while the Fe XII 193 line is more sensitive to the hotter plasma near the boundaries. For model 2, the temperature in and around the loop does not deviate much from the initial temperature throughout the simulation, which is close to the formation temperature of the Fe IX line. Therefore, for that model, we only use the Fe IX line for the forward modeling. Henceforward, we will refer to the Fe IX 171 line as the core line and the Fe XII 193 line as the boundary line.

We define the LOS angle such that $0^{\circ}$ is parallel to the positive $y$-axis and $90^{\circ}$ is parallel to the positive $x$-axis, which is the axis of oscillation. This is shown schematically in Figure 1. For correct comparison with observations for a given instrument with resolving power of $\mathrm{X}$ (which is defined as the FWHM of the point-spread function [PSF]; we take the plate- 
scale equal to half the spatial resolution unless otherwise explicitly stated), we degrade the original spatial resolution of the numerical model by first convolving the image of interest with a Gaussian with FWHM of X. We then resample the data according to the specific pixel size of the target instrument and add photon noise (Poisson distributed).

In the forward modeling, we have also considered various spectral and temporal resolutions. For a given spectral resolution, we convolve the numerical results with a PSF in the spectral dimension of the same size. We then resample the numerical results in the spectral and temporal dimensions with the target spectral resolution and cadence. Besides adding photon noise, we also add a 5\% random fluctuation at each wavelength position over the profile.

Unlike model 1, for which the ambient coronal environment has little emissivity in the selected emission lines, model 2 suffers from background emission from plasma at rest, due to the similar temperature inside and outside the flux tube. We therefore opt to subtract this background contribution prior to analysis by first eliminating the effect of having a square numerical box, which makes different LOS rays have different lengths. For this, we add artificial pixels with the same emission as a pixel with a background profile at rest, so that all LOS rays have equal lengths. Background subtraction is subsequently performed on every pixel, which now acts equally along any LOS.

\subsection{Imaging Signatures}

Figure 5 shows the time-distance diagram of the intensity in Fe IX 171 (for models 1 and 2) and Fe XII 193 (only for model 1) for an artificial slit placed transverse to the loop at its apex and for a LOS angle of $45^{\circ}$ (see Section 4.1 for the convention on the LOS angle). Since the Fe IX 171 line is more tuned to detect the plasma response at the core of the loop in model 1, while the Fe XII 193 line is more sensitive to the hotter plasma near the boundaries, we can see that the loop diameter is slightly larger in the boundary line at time $t=0$ as well as throughout the evolution. Correspondingly, as we will see clearly later on, the width of the loop in model 2 is wider than that in model 1.

Bright oscillatory strand-like structures are noticeable in both emission lines and in both models after about two periods, which correspond to the formation of TWIKH rolls. The amount of strand-like structure is largest in the boundary line of model 1. In the core line, we can see that the loop in model 2 shows a significantly larger amount of this fine structure than the loop in model 1 , as the uniform temperature of model 2 captures the TWIKH rolls in the cooler 171 line.

Although the overall damping is clear in Fe IX 171 for both models, the inner loop structure in the Fe XII 193 emission appears "decay-less," as explained in Antolin et al. (2016).

Another clear difference in the time-distance diagrams of Figure 5 between the core and boundary lines is the presence of periodic intensity brightening in only the boundary line near the loop boundary after KHI onset. This periodic brightening is further seen to extend over time in Figure 5, at first lasting under a minute and being almost continuous by the end of the simulation. In the first two periods prior to KHI onset, a small periodic brightening can also be seen in the boundary line in the trailing edge of the loop close to times of maximum displacement.
While the loop is observed to become thinner and dimmer in time for model 1 in Fe IX 171, the loop in Fe XII 193 becomes broader and brighter. The brightness increase and broadening occurs rapidly, in about one period after the onset of the KHI. In contrast, the intensity in Fe IX 171 in model 2 seems roughly constant on average. In Figure 6 for model 1 and in Figure 7 for model 2, we plot the evolution of the integrated intensity over the slit. In model 1 (top panel of Figure 6), we can see a clear intensity enhancement in the boundary line occurring right after the onset of the KHI with a steep linear increase over 2 periods up to a factor of 1.8 , followed by a gradual increase up to a factor of 2 in the remainder of the simulation. On the other hand, the core line in the same model shows dimming to a weaker degree, but also in a two-step behavior, leading to an overall decrease of $20 \%$. The trend observed in the same line for model 2 is very similar to that of model 1 , but with a much smaller magnitude of only $3 \%-4 \%$. This is because of the competing effect from adiabatic heating and cooling within the shell, as seen in Figure 4.

Similarly, on a relatively short timescale of about two periods after the KHI onset, the loop width increases, as shown in Figure 2. This evolution can be seen in Figure 6 for model 1 and in Figure 7 for model 2, even for low spatial resolution. After KHI onset, the loop width increases for the boundary line up to $17 \%-24 \%$, while it decreases for the core line by a factor of $11 \%-15 \%$. The variation in loop width for model 2 in the core line looks similar to that of the boundary line in model 1 . Indeed, although with a lower amplitude of only $3 \%$, a general increase in loop width can be seen in the third panel of Figure 7 , but only for high spatial resolution (below $0.5 R$ ).

The small-scale, periodic brightening near the loop boundary has an impact on the overall intensity. Indeed, by summing the intensity across the flux tube and subtracting the (smoothed out) general trend of the intensity evolution, we can discern multiple small oscillations in the intensity with maximums of about $1.5 \%$ and $0.2 \%$ for Fe XII and Fe IX, respectively, for model 1 , and $0.1 \%$ in Fe IX for model 2 . This is shown in the second panel from the top in Figures 6 and 7. The intensity oscillations in both lines in model 1 are initially in-phase and then go out-of-phase as soon as KHI sets in, which is suggestive of different mechanisms. The main intensity peaks in the boundary line are generally reached at times of maximum displacement (minimum velocity shear) and minimum displacement (maximum velocity shear) for the core line. The time locations of these peaks are independent of the LOS.

Periodic oscillations in the width of the loop can also be seen for any line and model. At high resolution, the loop width variation over half a period can be as high as $25 \%$, but decreases to $3 \%$ for a low spatial resolution of $1 R$. This effect has also been described in Yuan \& Van Doorsselaere (2016), although the origin of this behavior is different in both studies. The bottom two panels in Figure 6 for model 1 and in Figure 7 for model 2 show the evolution of the loop width for different spatial resolutions and for different LOS angles. Oscillations in the loop width can be seen with double the periodicity of the kink mode, in-phase between both lines prior to and following the KHI, although with some scatter.

Prior to KHI onset, these intensity (and loop width) oscillations are about a factor of five smaller, as is expected due to the highly incompressible nature of the kink mode (Van Doorsselaere et al. 2008a; Goossens et al. 2012). The small oscillations in intensity obtained during this time window are 

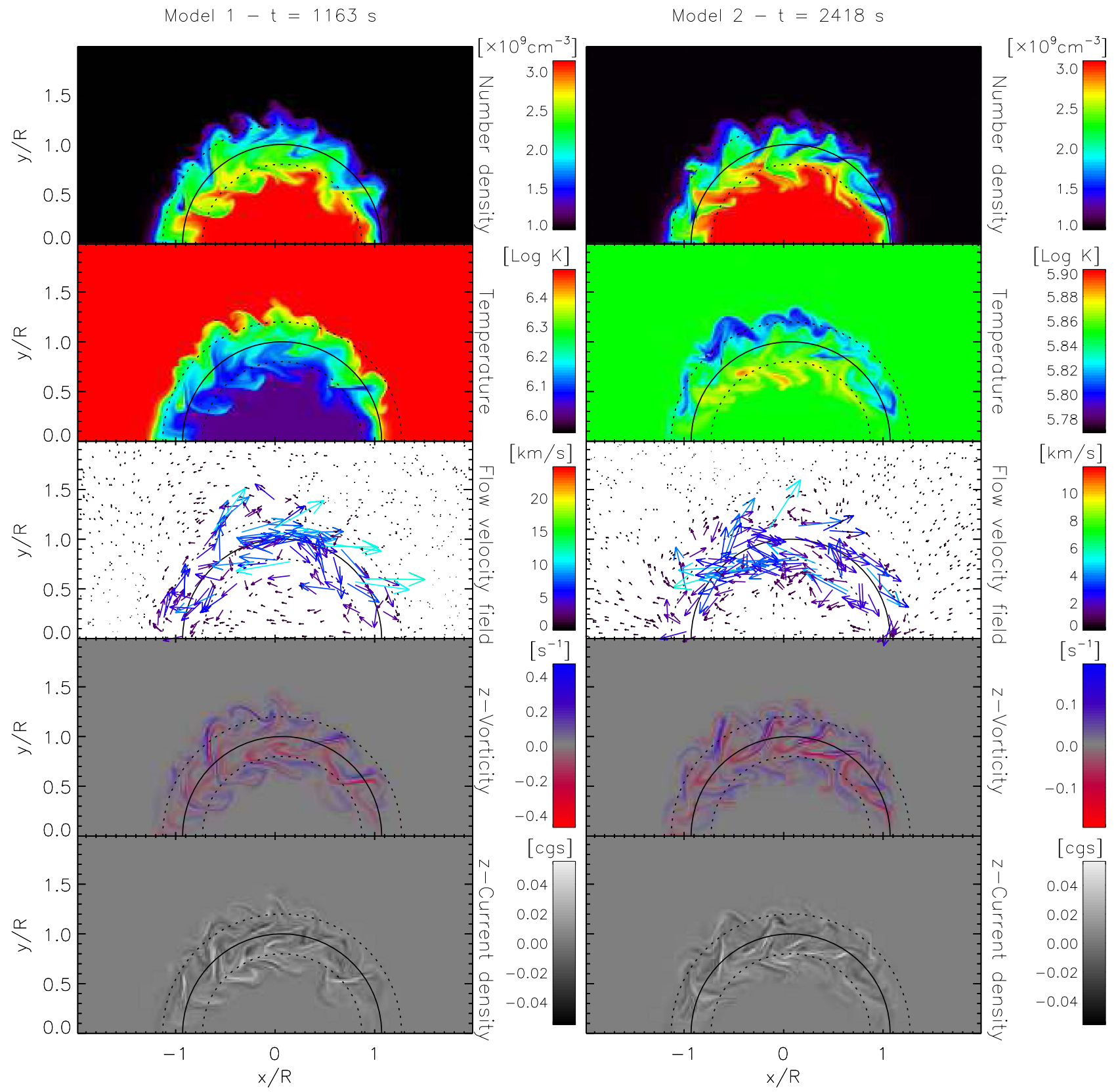

Figure 4. Snapshots of the simulations in model 1 (left panels) and model 2 (right panels) after roughly 4 periods. From top to bottom we have the number density, temperature (logarithm), flow velocity field, and the longitudinal (z-) components of the vorticity and current density. We overlay in black the initial radius of the loop (solid curve) and the minimum and maximum extent of the boundary layer (dashed curves).

(An animation of this figure is available.)

partly due to the quadrupolar terms in the wave solution characteristic of the kink mode, as described by Yuan \& Van Doorsselaere (2016), to a minor degree due to resonant absorption (which redistributes material in the boundary layer), and primarily due to the deformation of the flux tube from the combined effect of the inertia and fluting modes (Ruderman et al. 2010), both of which produce an ellipse with a maximum axis along the $90^{\circ} \mathrm{LOS}$ at times of maximum displacement. All of these effects impact the column depth (and are therefore dependent of the LOS angle) in a similar way for both lines, which explains the in-phase behavior.

The main features in Figures 6 and 7 are due to the TWIKH rolls, as discussed in Section 3.2. For model 1, the dominant effect of the TWIKH rolls is the mixing of internal and external plasma. This produces an increase of emission in the boundary line and a corresponding decrease in the core line, since the temperature of the plasma in the vortices shifts away from the maximum formation temperature of the core line and closer to that of the boundary line, thereby explaining the anti-phase behavior in the detrended intensity oscillation and the overall increase and decrease in the boundary and core lines, respectively. For model 2 , the intensity amplitude oscillations are mostly due to the compressive effect of the TWIKH rolls and are smaller than in model 1. TWIKH rolls in this model generally achieve their greatest size at times of zero displacement. Furthermore, a vortex forming at the trailing side of the 

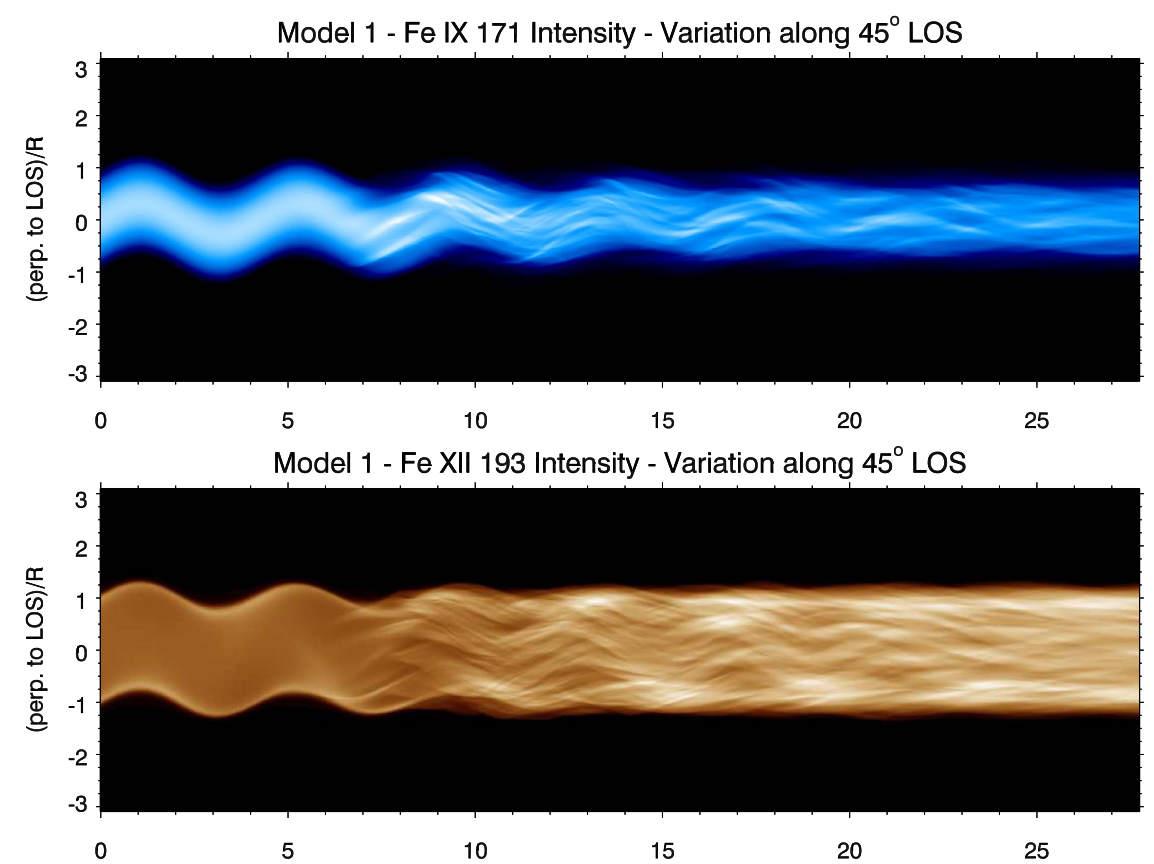

Model 2 - Fe IX 171 Intensity - Variation along $45^{\circ}$ LOS

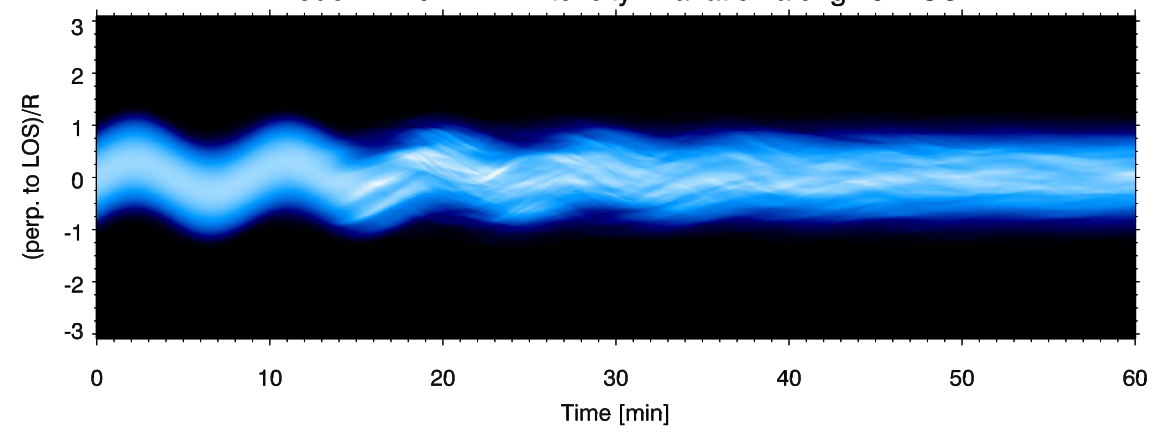

Figure 5. Time-distance diagrams of the intensities in the Fe IX (top panel) and Fe XII (middle panel) lines of model 1 and Fe IX line (bottom panel) of model 2 for a slit placed perpendicularly to the loop at the apex and with a LOS angle of $45^{\circ}$ and at numerical (highest) spatial resolution.

loop will be carried on top of the moving loop on the way back. Therefore, the vortex will be further away from the plane of oscillation at times of zero displacement, thus increasing the loop width (particularly for a LOS of $90^{\circ}$ ) and leading mostly to an in-phase modulation in both spectral lines. This effect also makes the loop width variation dependent on the LOS angle, as can be seen in Figures 6 and 7 (check the out-of-phase behavior between the $0^{\circ}$ LOS case and the $90^{\circ}$ LOS case for both lines). This behavior is not obtained when considering only the linear (quadrupolar) terms of the wave equation (Yuan \& Van Doorsselaere 2016).

Figure 8 shows the wavelet analysis for the detrended intensity time series for both lines in model 1 and the core line in model 2, where we can see the prevalence of mainly two periodicities: one at the global kink period (roughly $4.5 \mathrm{~min}$ and 9 min in models 1 and 2, respectively) and a similar or stronger peak at half that periodicity appearing when the KHI sets in. The power is also more extended in time for the boundary line in model 1 and the core line in model 2 . Interestingly, the power of the peak corresponding to the global kink mode is wider in frequency in model 1 for both lines, having roughly twice the width of the strongest peak at half the period. On the other hand, the width of both peaks in model 2 is comparable.

\subsection{Spectral Signatures: Phase Drifts and Ragged Transitions}

Many of the observed features in imaging data can be further understood from the spectral diagnostics. Figure 9 shows the Doppler velocity in both emission lines in model 1 and in the core line for model 2 for the numerical resolution and a LOS of $45^{\circ}$. The initial blue- and redshifts at the center of the flux tube correspond to the global kink mode, and this Doppler oscillation is roughly $90^{\circ}$ out-of-phase with the transverse (plane-of-the-sky, POS) motion, as expected (Goossens et al. 2014). At the loop boundary, corresponding to the top and bottom (thin) boundary regions in the figure, alternating thin "envelopes" of opposite Doppler shift with respect to the core are observed immediately from the start of the simulation. These initial thin envelopes correspond to the response of the external plasma to the kink mode, which moves azimuthally around the flux tube in the opposite direction (dipole return flows). It is worth specifying that the calculation of the Doppler component over the spectral profile has been performed at locations with an intensity above $15 \%$ that of the maximum initial intensity. For model 2, where the external and internal plasma have the same temperature, we have opted to subtract the background contribution from the plasma at rest, as described in Section 4.1. This procedure also affects the 
Model 1

Intensity variation for $\mathrm{a} 00^{\circ}$ LOS angle
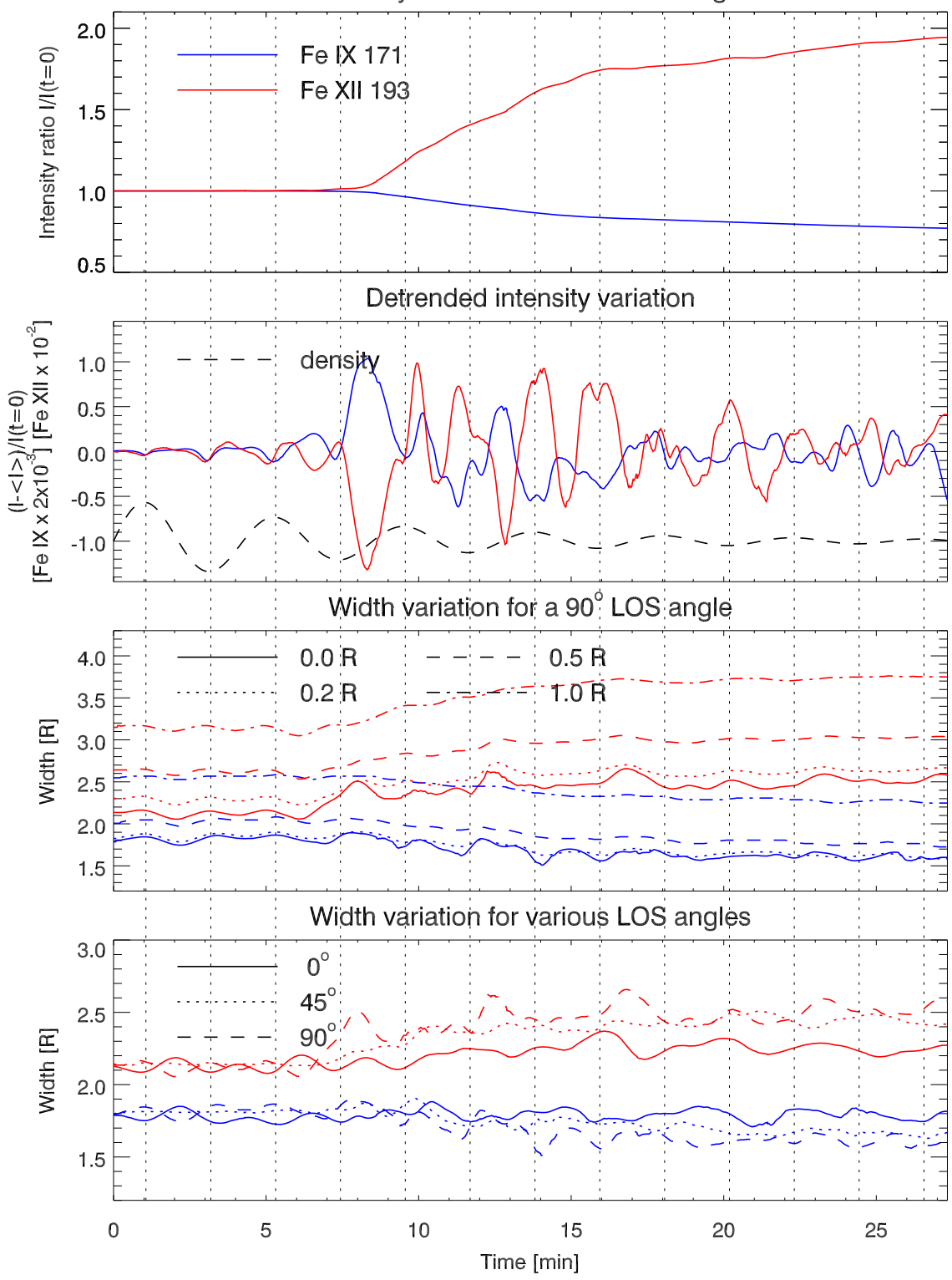

Figure 6. For all panels we show in red and blue the temporal variation of the quantities associated with the Fe IX and Fe XII lines, respectively. Top panel: variation of the intensity integrated over a slit placed perpendicular to the loop at the apex and at a LOS angle of $0^{\circ}$. Second panel from top: Detrended and normalized intensity shown in the top panel (note that the Fe IX intensity has been multiplied by 5 to fit in the same scale). The fit to the density is overlaid with a dashed curve with an adhoc scale. The fit is performed by first degrading the spatial resolution to $1 R$ for the density cross-section at the (previously defined) slit location, then fitting a Gaussian profile at each time (from which the centroid is calculated), and fitting an exponentially damped cosine to the centroid locations. Third panel from top: variation of loop width calculated from each intensity line for a slit placed perpendicular to the loop at the apex, at an angle of $90^{\circ}$ and for different spatial resolutions. The loop boundaries are defined by the locations where the loop intensity increases above $20 \%$ of the initial maximum intensity in each line. Bottom panel: variation of the loop width for different LOS angles and for a spatial resolution of $0 R$ (highest). The vertical dotted lines correspond to the extrema of the density fit oscillation.

intensity of the features at the boundary of the flux tube, thereby reducing the contribution from the quadrupolar terms. Correspondingly, the thin envelopes in Figure 9 are much reduced for model 2. Also, this procedure reduces the maximum detected Doppler velocity for that model, which should be similar to or larger than the initial kink amplitude.

Over the first two periods, an increase of the Doppler velocity amplitude in the thin envelopes (the loop boundary) is clearly seen in both lines and both models. This effect corresponds to resonant absorption. Accordingly, the largest Doppler components are the azimuthal resonant flows at the boundary of the flux tube, which are, however, confined to the very thin boundary layers until that moment (and therefore confined to regions of low emissivity, especially in the core line). When KHI sets in, vortices are generated around the boundary which carry similar characteristics as the resonant flow, especially its magnitude.

The direction of motion of the vortices will also be partly defined by the direction of the resonant flow. Initially, the TWIKH rolls move opposite to the loop core, as can be seen in Figure 3. This produces the observed jagged, irregular transitions between the blueshift and the redshift regions seen 
Model 2
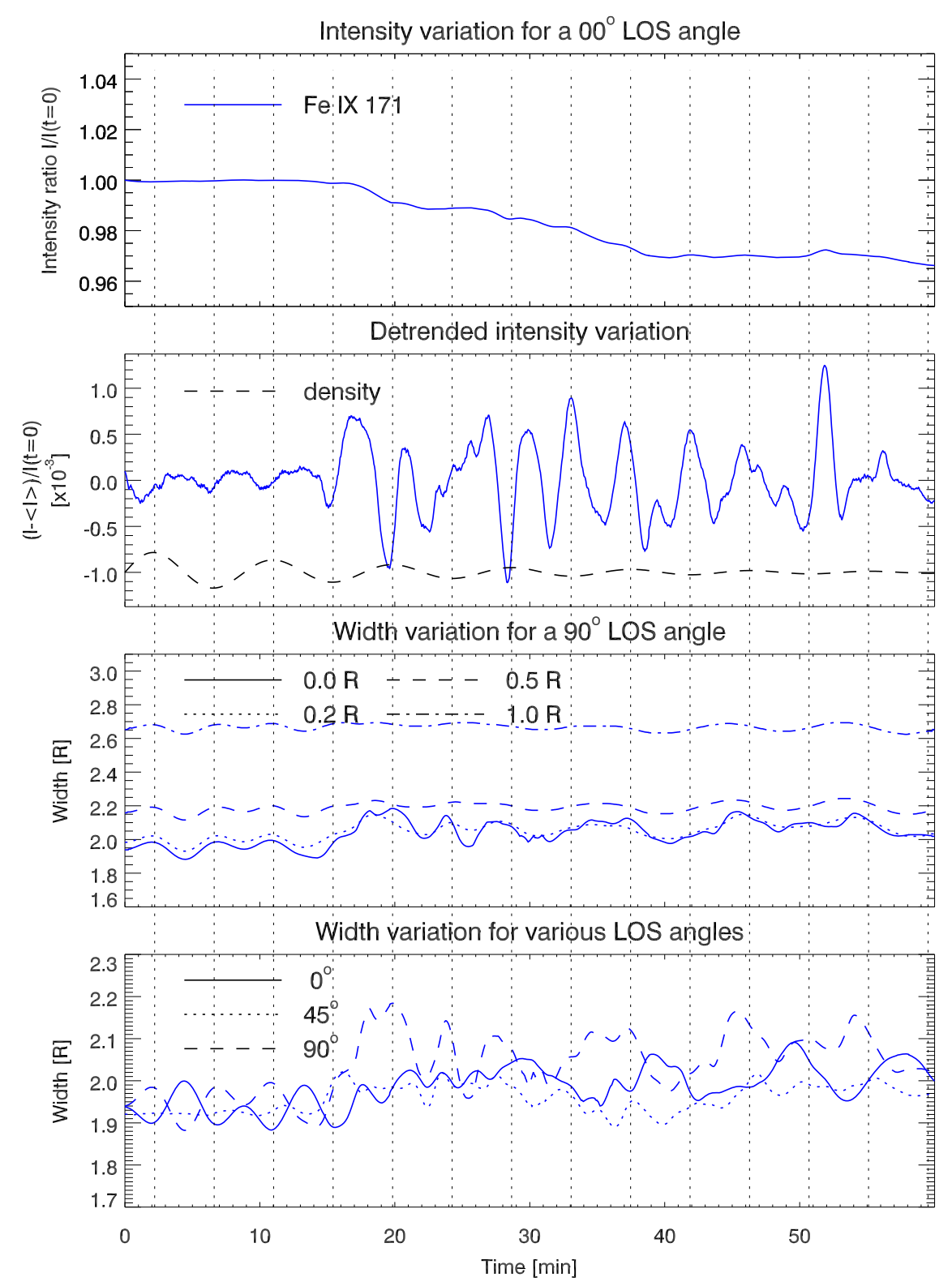

Figure 7. Similar to Figure 6, but for the Fe IX detrended intensity of model 2.

in both the core and boundary lines in Figure 9 where, in many locations, the blue- and redshifts appear interwoven. As the vortices grow, their crests continue moving with the resonant flow opposite to the loop core, while their troughs now move in the same direction as the loop core. This motion leads partly to the observed criss-crossing of features in Figure 5, and also leads to the generation of the next set of vortices (see the middle panel of Figure 3). Finally, when the crests break, they have maximum emissivity (especially in the boundary line) and now have the largest (azimuthal) velocity amplitudes. This effect produces a net $90^{\circ}-180^{\circ}$ phase difference between the POS motion and the LOS velocity. This effect was initially reported in Antolin et al. (2015) for the prominence case. Such a phase difference has also been observed with IRIS during a flare by Brannon et al. (2015), providing an alternative interpretation in terms of TWIKH rolls. The significant amplitude of the vortices also produces a filamentary structure in the Doppler images of Figure 9. This is especially clear in the boundary line, since in model 1 the vortices are on average hotter than the loop core.

The vortices rapidly break up into smaller vortices, while new vortices emerge and become dominant. The emergence of new vortices on top of old ones (moving in different directions), combined with the damping of the kink mode, produces a fading of the Doppler signal toward the loop center. This fading is not observed toward the edges. Since the LOS is tangential to the resonant flow there, most vortices along that LOS move coherently and with increasing amplitude from the resonance.

As the loop boundary is broadened due to the vortices, the density transition, and hence the change in phase speed, between the core and the external medium becomes flatter. This can be seen as arrow-shaped structures in the Doppler images, which become flatter as time passes due to phase mixing. 

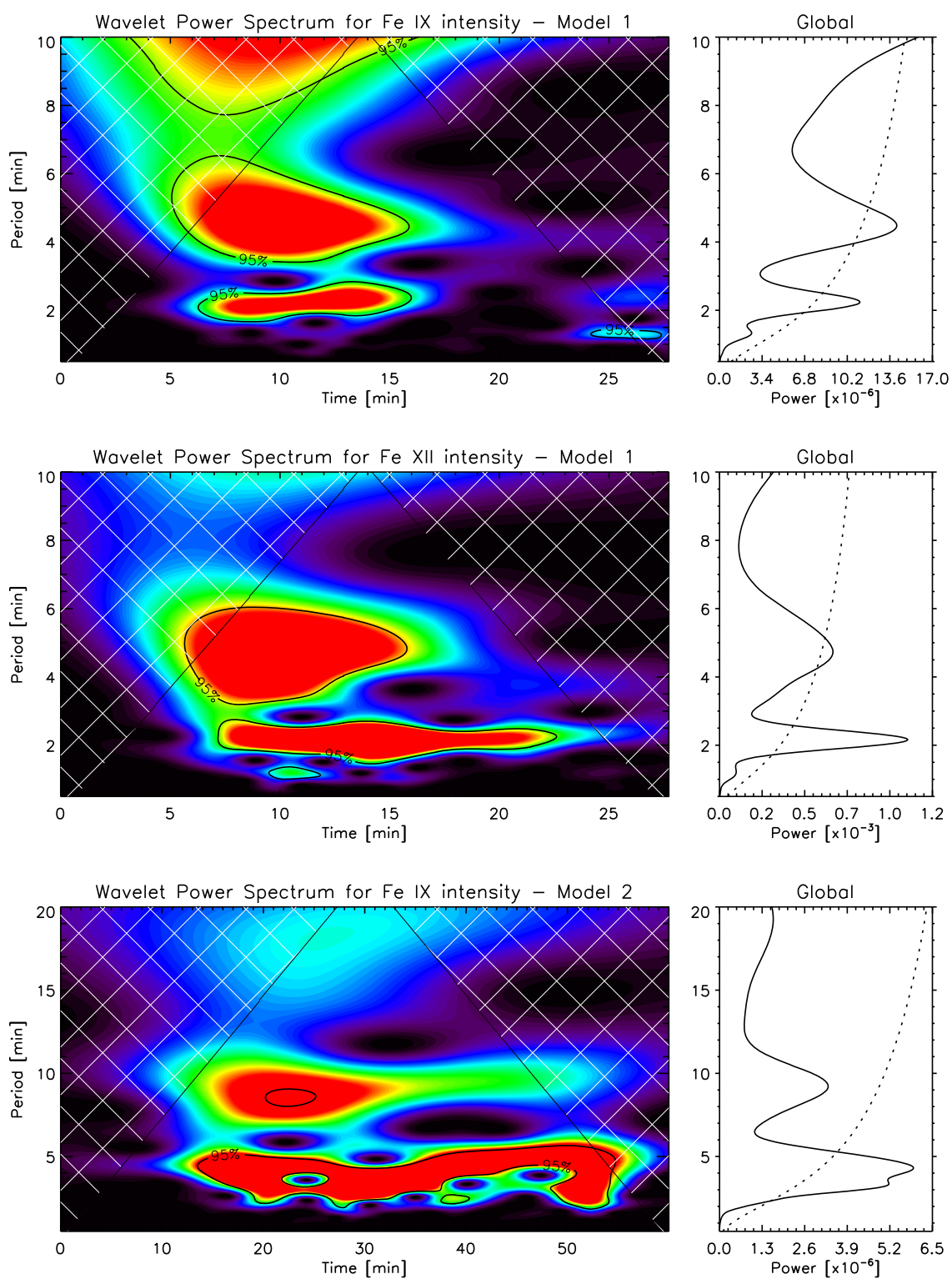

Figure 8. Wavelet analysis for Fe IX (top panel) and Fe XII (middle panel) detrended intensity lines in model 1 and Fe IX (bottom panel) in model 2 (computed with the Torrence \& Compo 1998 software). The significance level shown with thick contours encloses regions of greater than 95\% confidence, assuming a red-noise process as background spectrum (we estimate the lag-1 coefficient of 0.9). Cross-hatched regions indicate the cone of influence where edge effects become important.

\subsection{Line Broadening: Boundary Enhancement}

Line broadening (including both the thermal and nonthermal components) is also relevant for detecting the signatures of phase mixing (and resonant absorption), the formation of vortices, and the changes in temperature. When observing at high resolution, line broadening due to the higher external temperature appears at the start in model 1 for both the boundary and core lines as a significant increase of $5 \mathrm{~km} \mathrm{~s}^{-1}$ from the loop core (around 13-14 $\mathrm{km} \mathrm{s}^{-1}$ ) to the edges (around $18-20 \mathrm{~km} \mathrm{~s}^{-1}$ ). This is shown in Figure 10 (upper and middle panels). Accordingly, in model 2, this increase is largely absent at the beginning.

The TWIKH rolls can be clearly distinguished in the line broadening maps in both lines and models as a rapid enhancement around the loop edges, after about one period following the onset of KHI. This enhancement is stronger in model 1 where it appears uniform over time and all periodicity seems to be lost. This enhancement is produced by an increase in temperature evidenced by a decrease (increase) in line emission in the core (boundary) line, seen in Figure 5, and also by unresolved local motions along the LOS from the vortices. In model 2, for which the temperature is mostly uniform (and therefore only the non-thermal component is visible), the maps show a smaller range of variation, which is mostly due to the unresolved motions of the vortices and also from phase mixing. Indeed, the overlap of vortices producing the oscillatory behavior is a result of the overlap of azimuthal Alfvén waves at the boundary, which are continuously generated and in turn become unstable. Accordingly, we can see the formation of the vortices in these maps as periodic stretches of enhanced broadening of 1-2 $\mathrm{km} \mathrm{s}^{-1}$ that start at the loop edge, and extend toward the loop core. While model 1 shows an increase in the average line width in time for both lines after the onset of KHI, model 2 shows saturation followed by a small decrease. This difference between the models indicates that the main factor behind the long-term increase of the line widths in model 1 is 
Variation along $45^{\circ}$ LOS - Numerical resolution
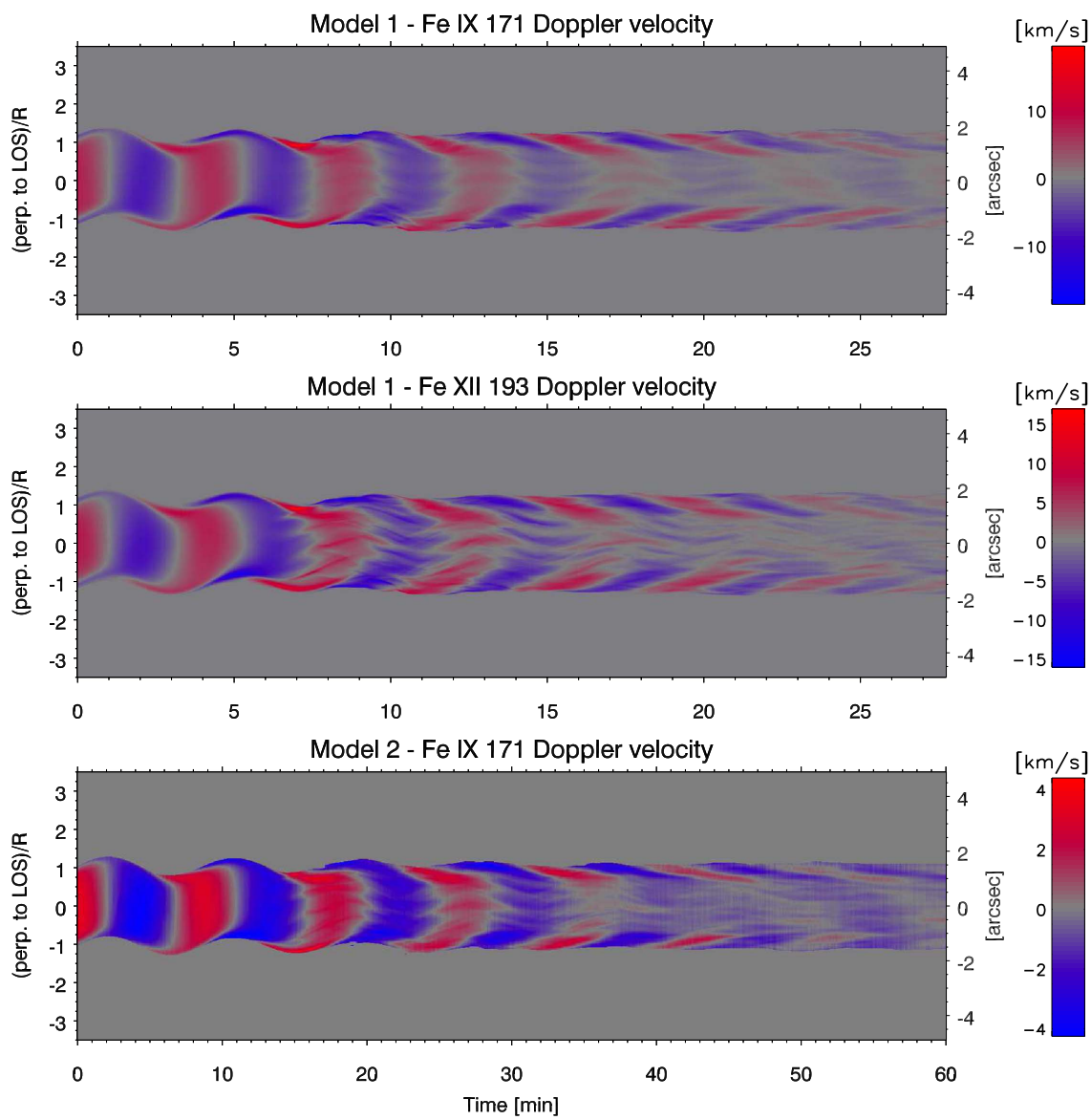

Figure 9. Doppler velocity in the Fe IX (top panel) and Fe XII (middle panel) lines of model 1 and the Fe IXline (bottom panel) for model 2 for a slit placed perpendicularly to the loop at the apex and with a LOS angle of $45^{\circ}$. The Doppler velocity is calculated by fitting a single Gaussian over the intensity profile for each pixel with an integrated intensity (over wavelength) above $15 \%$ of the maximum integrated intensity at $t=0$.

the thermal component rather than the non-thermal one, and is due to the mixing of plasma in the non-uniform temperature model.

In the first few oscillations, we see a periodic increase of the line broadening at the trailing edge of the loop at times of maximum displacement, which is more clearly visible for model 1 and for a LOS angle of $0^{\circ}$. At the same times in model 2, we can also see a small periodic increase of about $0.5 \mathrm{~km} \mathrm{~s}^{-1}$ across the loop (visible as slightly more yellow regions in the figure, due to the overall small line width variation in this model). These enhancements have double periodicity (with respect to the kink mode period) and are due to the deformation of the flux tube, which increases the column depth along the LOS and therefore the unresolved motions. After the onset of the KHI, this effect is altered due to the appearance of TWIKH rolls. The double periodicity remains but now the additional, dominating factor of the TWIKH rolls defines the phase relation with respect to the intensity. Since the TWIKH rolls appear mostly at times of maximum shear velocity but converge at the trailing edge of the loop, it makes the phase relation LOS dependent. We find that for a $0^{\circ} \mathrm{LOS}$, the intensity and line width are initially out of phase by $\pi$ for both the core and boundary lines (as also obtained by Yuan \& Van Doorsselaere 2016), meaning that the line width has maxima at times of maximum displacement. However, after
KHI, they become $\pi / 2$ out of phase only for the boundary line, with the line width peaking ahead of the intensity. This advance in the increase in line width seems to be due to the new set of oppositely directed vortices already appearing before reaching maximum displacement (see the online animated Figure 4), which are only picked out in the boundary line.

\section{Predicting Observable Features}

\subsection{Imaging Characteristics at Low Spatial Resolution and Different LOSs}

One of the main questions we want to address is which of the observational signatures described in the previous sections could actually be observed with the resolving power of current instruments, as well as with the next generation of detectors. We therefore look at the effect of degrading both the spatial and spectral resolution.

Degrading the temporal resolution has little effect on the results. This is because the timescale of most of the observed features, especially at lower spatial resolution, is on the order of half a period or one period ( 255 and $530 \mathrm{~s}$ for models 1 and 2, respectively), a timescale much longer than usual instrument cadences. However, the smallest scales in our model, which were obtained in the turbulent cascade of the TWIKH rolls, do have smaller timescales. However, their effect on the intensity 
Variation along $45^{\circ}$ LOS - Numerical resolution
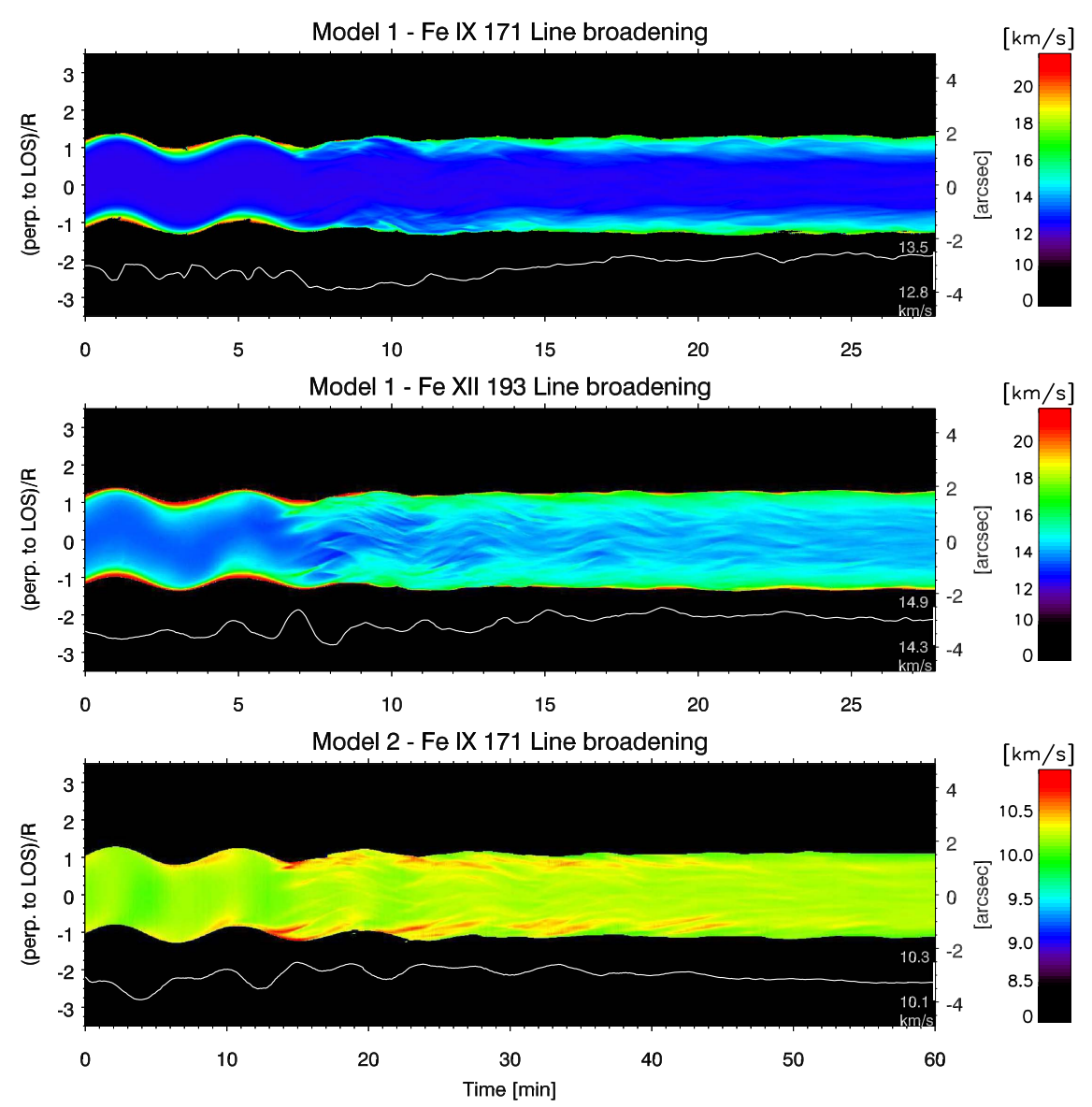

Figure 10. Similar to Figure 9, but for the line width instead of the Doppler velocity. Note that the color scale is shifted artificially toward higher values so that the range $0-8.5 \mathrm{~km} \mathrm{~s}^{-1}$ corresponds to black. The overlaid white curve corresponds to the average line width over non-zero values at each time step, smoothed over $14 \mathrm{~s}$ in order to bring out the main trend. The scale and minimum and maximum values of this average are written on the lower right side of each panel.

and Doppler velocity is minimal and contributes little to the line widths.

Figure 11 shows time-distance diagrams for both the core Fe IX 171 and boundary Fe XII 193 lines for a fixed LOS angle of $45^{\circ}$ mimicking an instrument such as $\mathrm{Hi}-\mathrm{C}$, with a spatial resolution of 0 ". 33 and $5 \mathrm{~s}$ cadence. We can see that the fine loop substructure is still visible in all of the lines and models, as well as the overall intensity trend of thinning/broadening and fading/brightening in the core/boundary lines, and the periodic intensity changes (particularly in the boundary line) produced by resonant absorption and the KHI. We now further degrade the spatial resolution to 1 !" 2 and the temporal resolution to $15 \mathrm{~s}$, representing $S D O / \mathrm{AIA}$, and show the results in Figure 12 for the same settings as in Figure 11. In this case, only the overall fading/brightening and the corresponding thinning/broadening is observed. The damping in the core line and decay-less oscillation in the boundary line can be clearly observed. The damping in the core line for both models looks very similar, with only a slightly longer damping time in model 2 compared to model 1 (with the damping time over period ratio being 2.36 and 2.30 , respectively).

The fact that the decay-less effect (described in detail in Antolin et al. 2016) is basically not observed for model 2 indicates that the temperature contrast between the loop and the ambient corona, combined with the mixing produced by $\mathrm{KHI}$, is an essential ingredient in order to obtain this effect. In theory, if the KHI can lead to efficient heating in a more realistic model (for instance, with a more realistic turbulent spectrum at higher spatial resolution and with the expected reconnection between the vortices), then we may expect the decay-less effect to occur even with initially uniform temperature cross-sections. This is simply because in such a model the vortices would be at a higher temperature, thereby achieving the same effect as in the boundary line of model 1 .

\subsection{Spectroscopic Characteristics at Low Spatial and Spectral Resolutions}

For spectral instruments, we have assumed coarser spatial resolutions of 0 ". 4 and $3^{\prime \prime}$ (and keeping the temporal resolutions of 5 and $15 \mathrm{~s}$ ). The latter is representative of the Hinode/EIS resolution by taking a plate-scale of $1^{\prime \prime}$ but a PSF of $3^{\prime \prime}$. We also take three different spectral resolutions of 3,25 , and $36 \mathrm{~km} \mathrm{~s}^{-1}$, with the latter being roughly that of Hinode/EIS for the wavelength range of the core and boundary lines considered here.

In Figures 13 and 14, we fix the spatial resolution to 0 " 4 and take spectral resolutions of $3 \mathrm{~km} \mathrm{~s}^{-1}$ and $25 \mathrm{~km} \mathrm{~s}^{-1}$, respectively. In each of these figures, we have overlaid the intensity contours at various levels (with the outermost being at $50 \%$ of the maximum intensity at $t=0$ ) corresponding to the same spatial resolution (and with the other characteristics as well, such as re-binning and photon noise). These contours indicate 

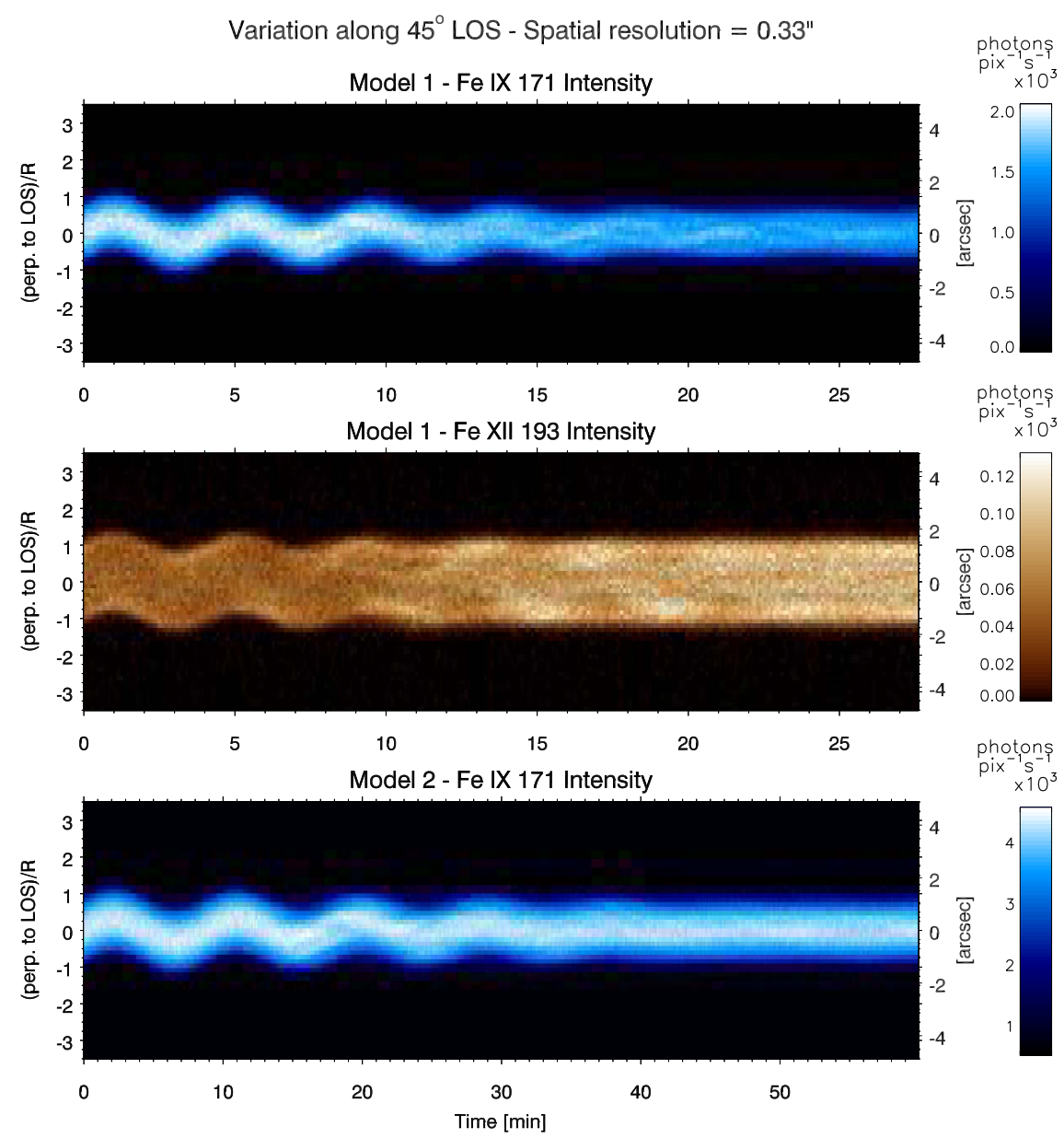

Figure 11. Time-distance diagrams for a slit in the same configuration as in Figure 5 for the loop of model 1 in the Fe IX (top panels) and Fe XII (bottom panels) intensity lines, but mimicking an imaging instrument such as Hi-C (with a spatial resolution of 0 ." 33 and a cadence of 5 s). Pixel sampling at half the resolution and $10 \%$ photon noise are taken into account. Model 2 seen in the Fe IX emission line and with the background emission subtracted.

which of the spectral features could actually be observed or suffer from instrument, LOS projection, and other effects. We notie that the fine structure in the Doppler signal (the ragged transition and single features from the vortices) mostly disappears, except for the case of the boundary line and only for time periods after the KHI onset. The arrow-shaped Doppler structure can also be seen in all lines and models at both spectral resolutions. However, in the core line of model 1, the arrow features are only detectable after the boundary has broadened due to KHI. At $25 \mathrm{~km} \mathrm{~s}^{-1}$ spectral resolution, the Doppler transitions and arrow-shaped features fade out rapidly and cannot be properly detected after five periods. The strongest Doppler signatures produced at the edge of the flux tube from resonant absorption are visible in the core line of model 1 only if the noise levels are less than roughly $15 \%$ of the initial maximum intensity. In the boundary line of model 1 or the core line of model 2, this condition is relaxed and the increase of amplitude can be detected even at pixels with noise levels as much as $50 \%$ the initial intensity. For the boundary line, this feature can be detected even at pixels with $90 \%$ of the initial intensity.

Figure 15 shows the Doppler signatures for an instrument like Hinode/EIS. In this case, the fine-scale structure completely disappears and the arrow-shaped Doppler profiles are only marginally visible in the boundary line at pixels with
$50 \%$ of the initial intensity. After four periods, the periodic Doppler change becomes hard to detect in all lines and models.

The combination of the intensity dimming/enhancement in the core/boundary lines with the arrow shape from phase mixing results in the Doppler velocity in the boundary line phasing out with respect to the Doppler velocity in the core line. This out-of-phase behavior is still detectable in coarse resolution instruments (see Figures 13-15).

It is important to note that at low spatial resolution, the maximum observable Doppler shifts at the boundary, which should actually be on the order of the initial amplitude of the kink mode (but can become significantly larger at small scales due to the resonance), are a factor of two smaller than the initial perturbation. This is important when we try to reconstruct the initial kink mode perturbation from observations and may explain the relatively small Doppler shifts from kink waves in non-flaring events detected with Hinode/EIS (Van Doorsselaere et al. 2008b). On the other hand, we obtain roughly the same Doppler range in Figures 13-15, indicating that lower spectral resolution does not have a significant effect on capturing the main features in the Doppler signals. This is because even with a low number of points across the spectrum, the line center can still be calculated fairly accurately from Gaussian fitting and centroiding.

Figures 16-18 show the corresponding line width measurements with the same settings as for the Doppler figures, that is, 


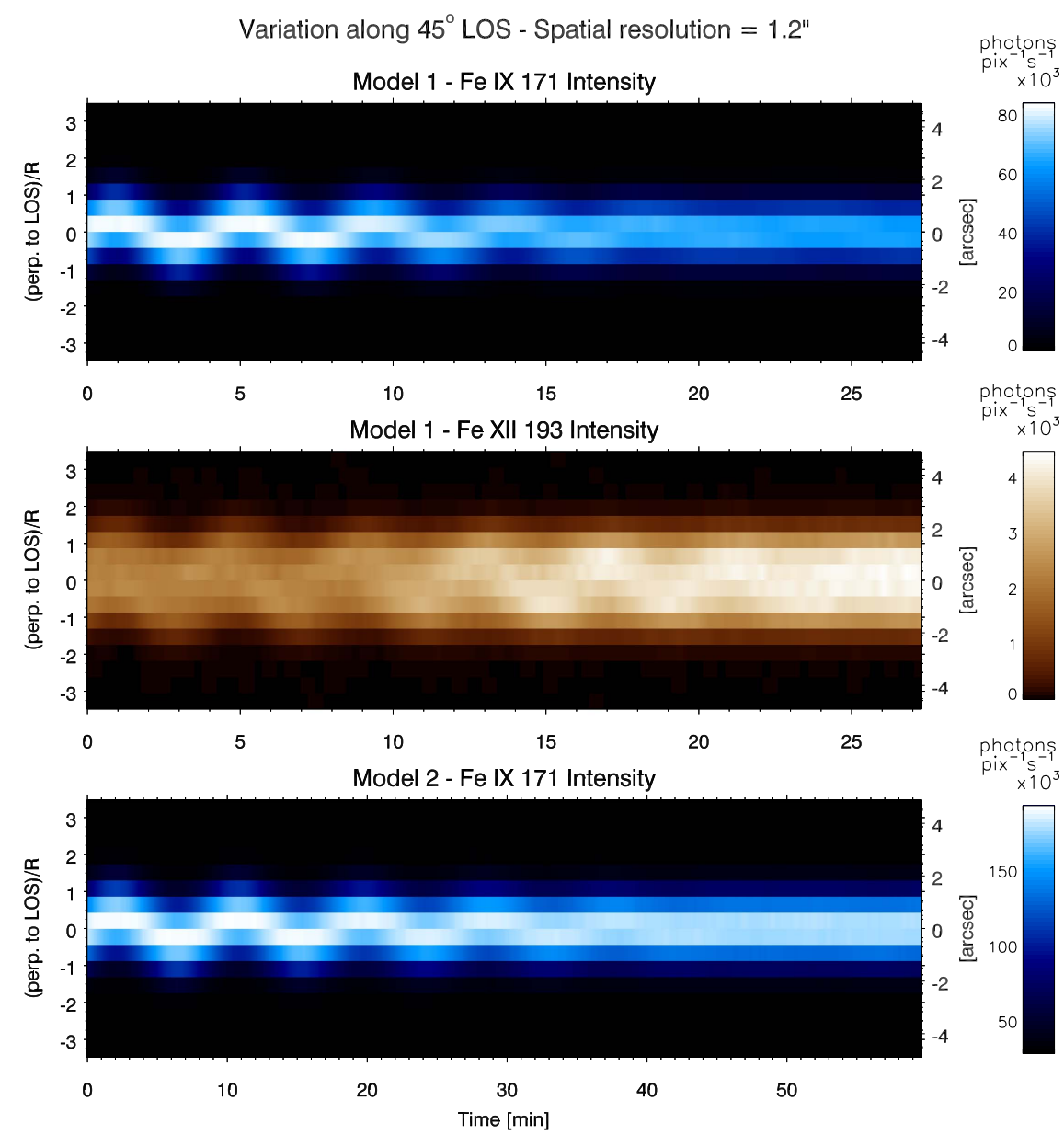

Figure 12. Same as Figure 11, but mimicking an instrument such as SDO/AIA (we take a spatial resolution of $1{ }^{\prime \prime} 2$ and a cadence of $15 \mathrm{~s}$ ).

Figures 13-15, respectively. At high spectral resolution, the line broadening at the loop edges can be seen in all lines and models, but particularly in model 1 . The $1-2 \mathrm{~km} \mathrm{~s}^{-1}$ enhancement from the non-thermal component is still visible in model 2, but its periodic variation is only visible for about two periods after the KHI onset. The change from pre to post-KHI is the most visible feature in model 1. At low spectral resolution, the line broadening enhancement at the edges in model 1 is still visible. However, the non-thermal component in model 2 is basically undetectable, indicating that the detected line broadening in model 1 is only from the combined thermal (from mixing) and non-thermal components. Even in model 1, the pre- to post-KHI change in line width is less clear at low spectral resolution.

At the low spatial and spectral resolution of Hinode/EIS, the pre- to post-KHI increase of line width can still be detected, but only in the core line of model 1 . Hence, the detectability of the line width between core and boundary appears better with a lower spatial resolution of $3^{\prime \prime}$, rather than $0 . " 4$. This is because the larger pixel size at the boundary captures a wider variety of unresolved motions which are then better picked up at high spectral resolution (especially when such unresolved plasma has lower intensity than the main component of the loop core). This may also explain why we cannot see the line width increase in the boundary line at low resolution, since the addition of plasma emission from the loop core (which is dynamically very different, thereby generating broader line widths) is much reduced in that line compared to the core line.
Furthermore, due to this effect but particularly because of the lower spectral resolution, an unrealistic increase of line widths in all lines and models is obtained, especially for model 2. When changing the spectral resolution from 3 to $25 \mathrm{~km} \mathrm{~s}^{-1}$, an almost double increase in the line widths is obtained and the line widths measured in the synthesized EIS spectra are roughly twice the true values measured in Figure 10.

\section{Discussion}

In our non-uniform temperature case of model 1, the loop is colder than the ambient corona. It is important to note that this model differs minimally from a model in which the loop is both hotter and denser than the ambient corona (but which presents a corresponding lower magnetic field strength in order to maintain pressure balance). Indeed, both the resonant properties and KHI threshold of the loop remain largely the same. By setting the ambient temperature to $1 \mathrm{MK}$ in such a model and the internal loop temperature to $1.5 \mathrm{MK}$, very similar forward modeling results would then be obtained with the same pair of emission lines, except that the results corresponding here to the core and boundary lines would be switched.

A series of physical properties have been identified leading to loop width, intensity, Doppler, and line width variations and specific phase relations, all summarized in Table 1 . In the present model, which includes non-linear effects, we have shown that the main factors are the combination of the KHI and resonant absorption (at least for the amplitudes considered here, 


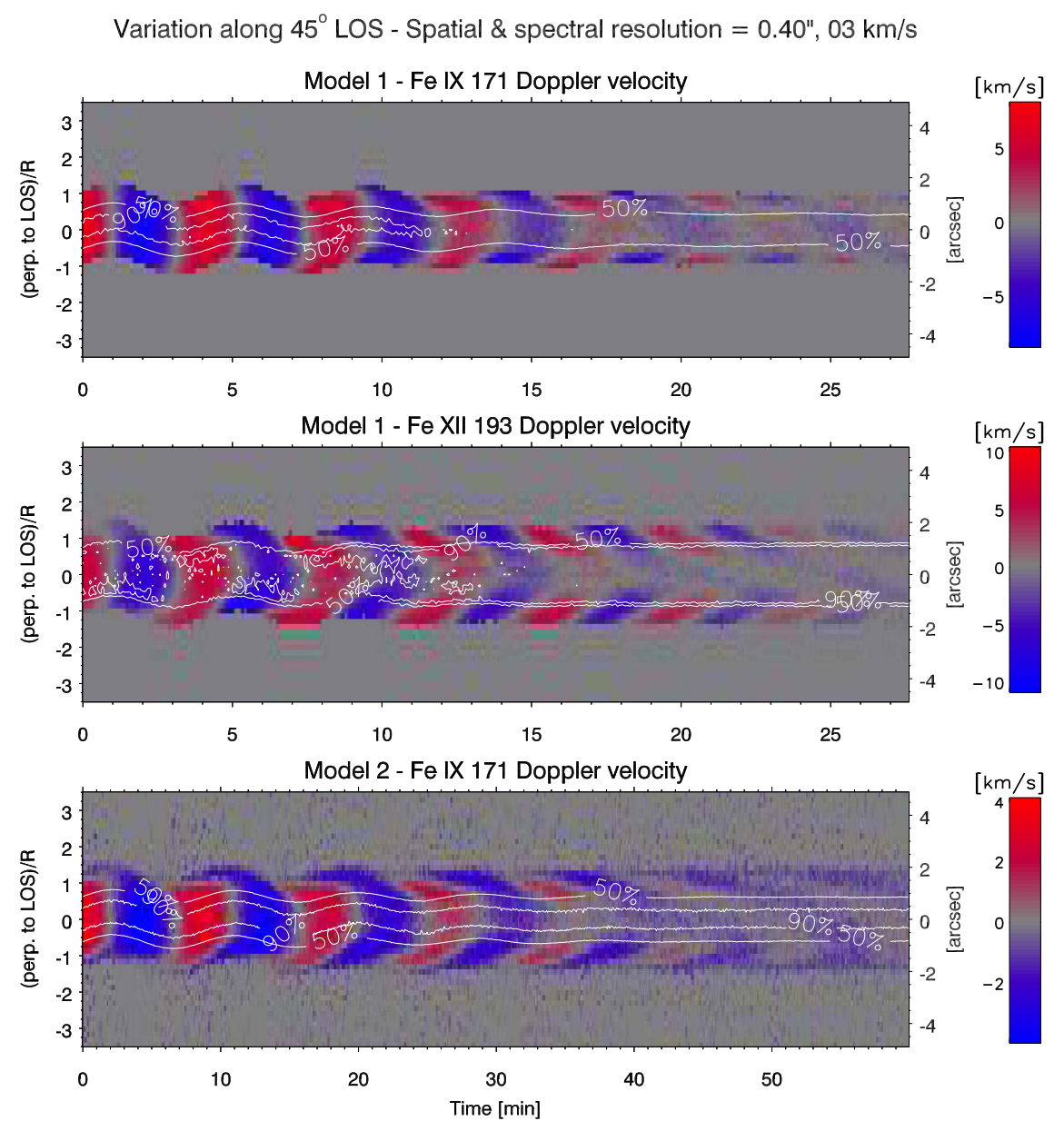

Figure 13. Similar to Figure 11, but for the Doppler velocity. The target instrument has a slightly different spatial resolution of 0 ". 4 and a spectral resolution of $3 \mathrm{~km} \mathrm{~s}^{-1}$. The intensity contours with the corresponding spatial resolution are overlaid in each diagram, enclosing regions with $50 \%$ and $90 \%$ intensity signal with respect to the maximum intensity at $t=0$.

which match currently observed, non-flare-related amplitudes), and to a minor degree the inertia and fluting modes produced by the initial kink mode. As shown in Antolin et al. (2015), the TWIKH rolls carry over characteristics of the resonant absorption and phase mixing mechanisms, allowing these to be detected at large, observable scales.

We find that the intensity variation in the core and boundary lines in model 1 are mostly due to the mixing between the interior and exterior plasma, which are at different temperatures. Indeed, the results from the uniform temperature model suggest that there is very little actual wave dissipation produced by the KHI in the current model, at least at the loop apex where the TWIKH rolls are bigger (and where therefore viscous dissipation is stronger) and where this study has focused. It remains an open question whether at higher resolution and different resistivity (for instance, with anomalous resistivity) efficient wave dissipation and overall heating is achieved ( $T$. Howson et al. 2017, in preparation; K. Karampelas et al. 2017, in preparation). The growth of the unstable modes is not the same along the loop, and the generated vortices are larger in amplitude close to the apex and smaller toward the footpoints. The difference in amplitude with height produces a local twist in the loop at the place of the roll-up. As the vortices degenerate into smaller and smaller structures, so does the local twist, which is reflected in multiple current sheets where neighboring magnetic field lines have a small but non-zero angle with each other. We hypothesize that component magnetic reconnection could occur in this configuration, leading to possible complex braiding on scales set by the TWIKH rolls, and to efficient heating.

In Antolin et al. (2016), we have shown that at a spatial resolution corresponding to AIA, the detected damping can vary depending on the emission line for loops with nonuniform temperature cross-section, which can lead to out-ofphase behavior between the boundary and core lines. For a model with a cold core, hotter emission lines will observe shorter periods. This effect is produced by phase mixing and the temperature gradient in the boundary, which allows a temperature specific channel (such as that of AIA 171) to pick up the azimuthal Alfvén waves that have a particular period in the boundary layer corresponding to that temperature. Such a temperature-dependent difference in phase can therefore be used to obtain an estimate of the local density in the boundary layer for which the emission line is strongest. MHD seismology could be performed to investigate the density inhomogeneity in coronal loops with non-uniform temperature cross-section. Correspondingly, here we have shown that the same effect leads to out-of-phase behavior between the Doppler velocities of both lines. Also, the wavelet analysis of the intensity modulation indicates a broad power peak at the kink period compared to a thin, stronger power peak at half that period (produced mainly by KHI) only for the non-uniform 


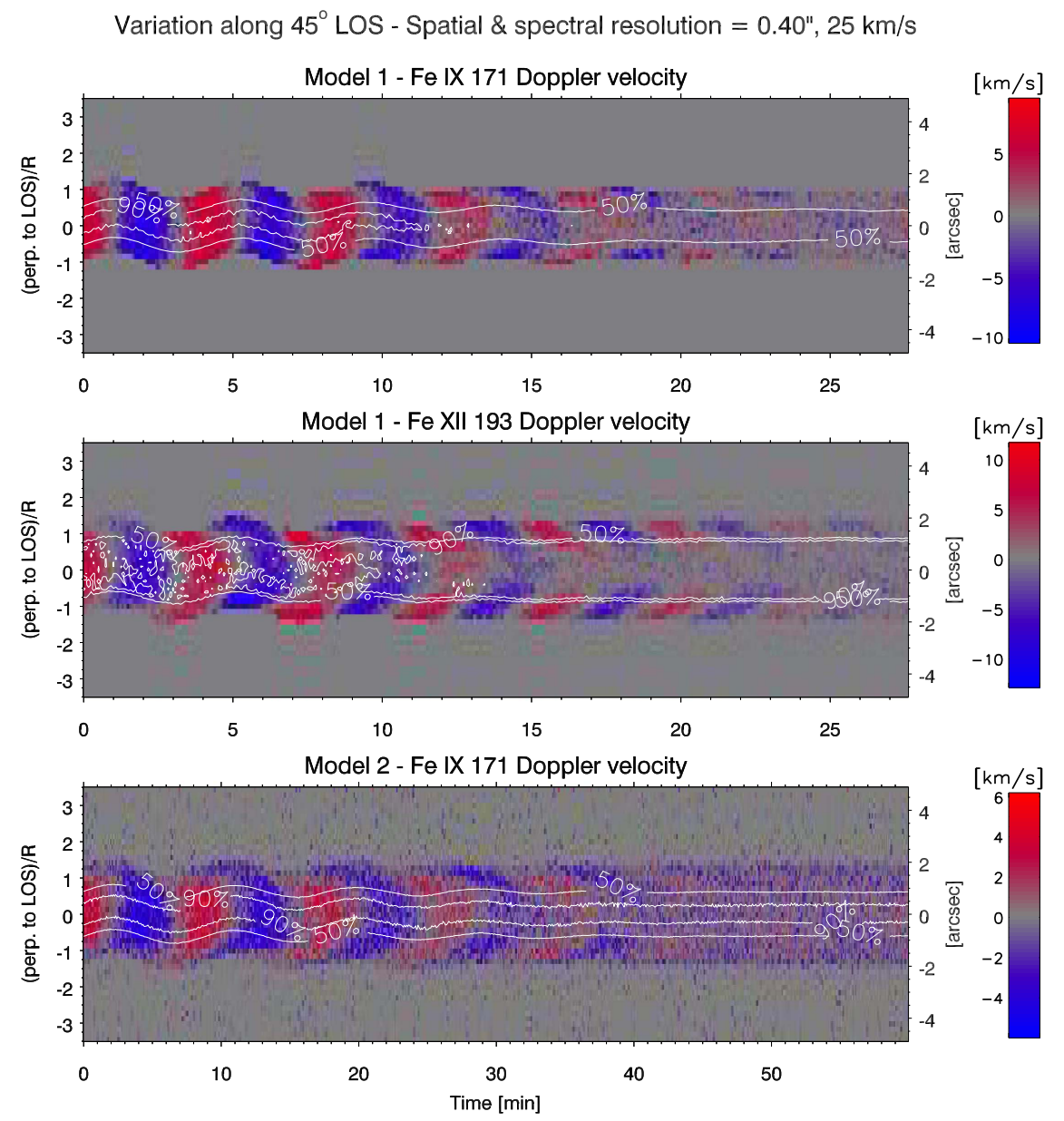

Figure 14. Similar to Figure 13, but with a spectral resolution of $25 \mathrm{~km} \mathrm{~s}^{-1}$.

temperature model. Since phase mixing depends only on the density gradient and is thus the same in both models, this effect is purely due to temperature. In the uniform temperature model, the 171 line picks up the intensity changes produced by the TWIKH rolls over a broader region encompassing both the core and the boundary, therefore leading to a broader power peak at half the kink period. Wavelet analysis could therefore be used as a diagnostic of temperature variation in the loop cross-section.

For spectral instruments, the Doppler variation and line width shown by both the core and boundary lines in the nonuniform temperature model also present important differences linked to the main physical mechanisms. A characteristic arrow-shaped structure in time-distance diagrams is observed due to the combined effects of the KHI, resonant absorption and phase mixing. Resonant absorption strongly damps the Doppler shifts of the global kink mode (which is barely visible after 3 periods or so), transferring most of the power to the azimuthal Alfvén waves in the boundary layer. At the same time, the KHI broadens the boundary layer, the vortices carry the momentum of the resonant flow, producing a ragged Doppler transition (fine-scale structure) at high spatial resolution, and a maximum Doppler signal toward the edges of the flux tube which can dominate the overall signal. Phase mixing leads to the characteristic arrow-shaped structure, meaning that the Doppler velocity will show $\pi / 2-\pi$ phase difference with the transverse (plane-of-the-sky) displacement of the kink mode. This effect can be captured for a spectral resolution of $25 \mathrm{~km} \mathrm{~s}^{-1}$ and spatial resolution of 0 .' 33 , and is mostly lost for a coarse spatial (and spectral) resolution such as that of Hinode/EIS.

Lowering the spatial resolution to 0 ". 4 significantly reduces the maximum observed Doppler velocity by roughly $30 \%-$ $50 \%$. This is partly due to the localization of the large Doppler values to the boundary layer from resonant absorption, but also because of phase mixing, leading to the superposition of various layers of TWIKH rolls along the LOS. While the boundary layer expands due to KHI, thereby reducing the first effect, phase mixing increases in time leading to Doppler velocities of only a few $\mathrm{km} \mathrm{s}^{-1}$ by the end of the simulation. However, the results of the uniform model indicate that most of the wave energy is not dissipated and therefore remains in plasma motions and in the magnetic field. The estimation of the wave energy based on the observed Doppler velocities at the end of the simulation would therefore provide a very inaccurate value of about $10 \%$ the initial wave energy. It is interesting to note that a similar value is found in De Moortel \& Pascoe (2012) where the underestimation is due to mode coupling and LOS superposition of multiple flux tubes.

As explained in the introduction, an important question is how the true wave energy content of the solar corona can be determined observationally. Figure 19 shows the kinetic energy in each of our models, based on the observed Doppler velocities and non-thermal line widths, for the $45^{\circ}$ LOS and 


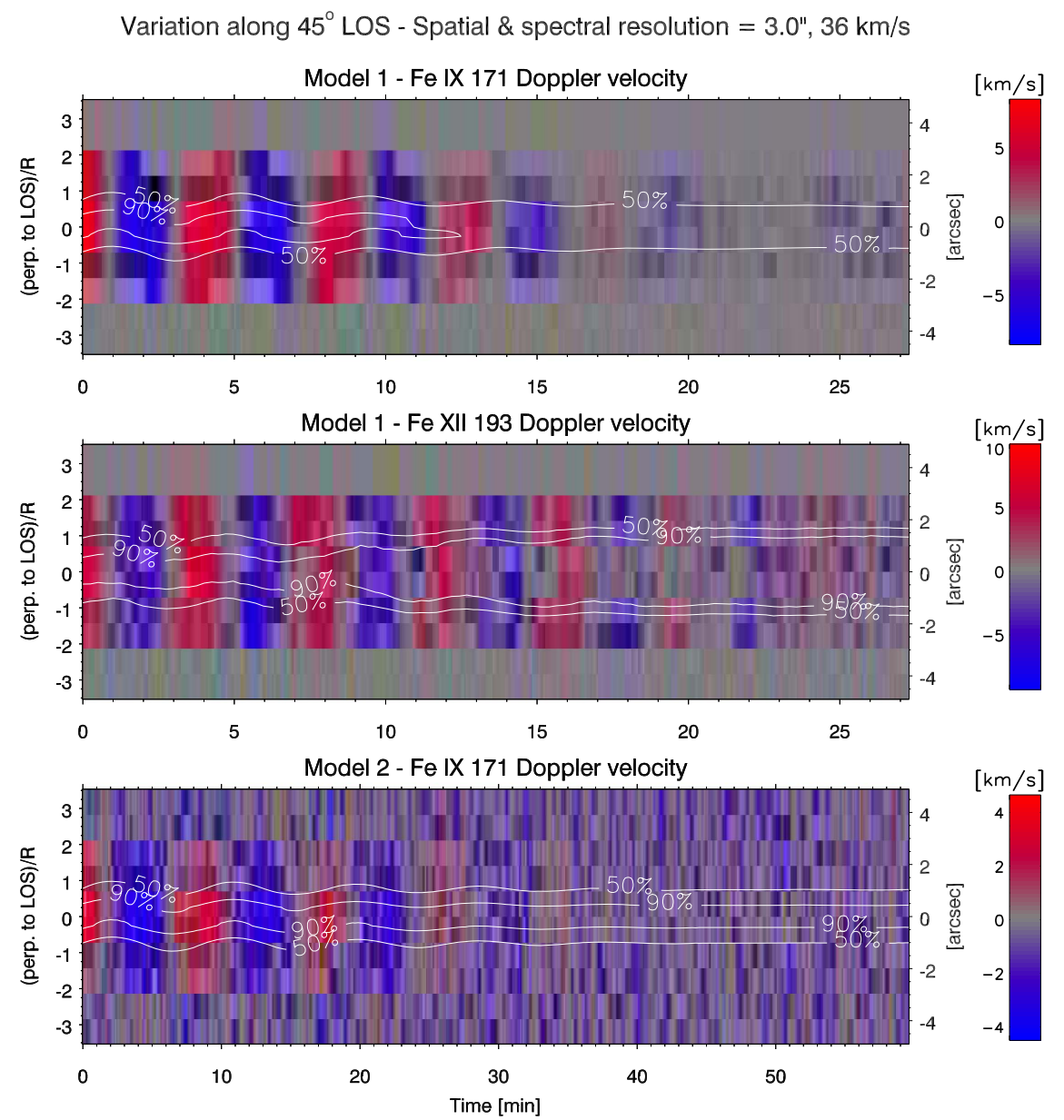

Figure 15. Similar to Figure 13, but with a spatial resolution of $3^{\prime \prime}$, a spectral resolution of $36 \mathrm{~km} \mathrm{~s}^{-1}$, and a temporal resolution of $15 \mathrm{~s}$, mimicking Hinode/EIS.

at full numerical resolution. The observed energy flux in a given spectral line integrated across the flux tube at the apex is calculated as follows:

$$
\begin{aligned}
F_{\mathrm{obs}}(t)= & \frac{1}{2} \int_{\perp \operatorname{LOS}}\left\langle\rho_{\lambda}(l, t)\right\rangle\left(v_{\mathrm{Dop}, \lambda}(l, t)^{2}\right. \\
& \left.+\xi_{\lambda}(l, t)^{2}-\xi_{\lambda, t h}(l, t)^{2}\right) c_{k} d l_{\perp},
\end{aligned}
$$

where the integration is over the perpendicular direction to the LOS, $c_{k}$ is the kink speed, $v_{\text {Dop }, \lambda}(l, t)$ is the Doppler velocity along a LOS ray $l$ and at a given time $t,\left\langle\rho_{\lambda}(l, t)\right\rangle$ is the emissivity-weighted average of the density along a LOS ray $l$ and at a given time $t$ :

$$
\left\langle\rho_{\lambda}(l, t)\right\rangle=\frac{\int_{l} \rho\left(l^{\prime}, t\right) \epsilon\left(l^{\prime}, t\right) d l^{\prime}}{\int_{l} \epsilon\left(l^{\prime}, t\right)} d l^{\prime},
$$

where $\epsilon(l, t)$ is the emissivity along the LOS ray $l$ and at a given time $t$. Also, $\xi_{\lambda}(l, t)$ and $\xi_{\lambda, t h}(l, t)$ are, respectively, the total line width in $\mathrm{km} \mathrm{s}^{-1}$ and the thermal component of the line width calculated with the emissivity-weighted average of the temperature along the LOS ray $l$ and at a given time $t$ :

$$
\left\langle T_{\lambda}(l, t)\right\rangle=\frac{\int_{l} T\left(l^{\prime}, t\right) \epsilon\left(l^{\prime}, t\right) d l^{\prime}}{\int_{l} \epsilon\left(l^{\prime}, t\right)} d l^{\prime},
$$

$$
\xi_{\lambda, t h}(l, t)=\frac{c}{\lambda_{0}} \sqrt{\frac{\left\langle T_{\lambda}(l, t)\right\rangle k_{B}}{\mu m_{p}}},
$$

where $c$ is the speed of light, $\lambda_{0}$ is the wavelength of the emitting element at rest, $k_{B}$ is the Boltzman constant, $m_{p}$ is the proton mass, and $\mu_{\lambda}$ is the atomic weight (in proton masses) of the emitting element.

In Figure 19, we also show the full kinetic energy flux in the same plane along the $45^{\circ} \mathrm{LOS}$, divided by the width of the flux tube $w \approx 2 R$ :

$$
\begin{aligned}
F_{\text {tot }}(t)= & \frac{c_{k}}{w} \int_{x} \int_{y} \frac{1}{2} \rho(x, y, t)\left(\left(v_{x}(x, y, t) \sin (\theta)\right)^{2}\right. \\
& \left.+\left(v_{y}(x, y, t) \cos (\theta)\right)^{2}\right) d x d y .
\end{aligned}
$$

The figure shows that the observed kinetic energy of the wave in both models and lines is roughly constant on average over the first few periods, after which it decreases by a factor of five by the end of the simulation, following the trend of the total kinetic energy. This behavior can be understood by the variation of the Doppler velocities and non-thermal line widths averaged across the flux tube. The former decreases due to resonant absorption, while the latter increases due to the KHI and phase mixing. As noted previously in Figure 10, the increase of the non-thermal component of the line width saturates about halfway through the simulation and remains roughly constant from thereon. This is because the motions from KHI degenerate into a turbulence-like regime from which 


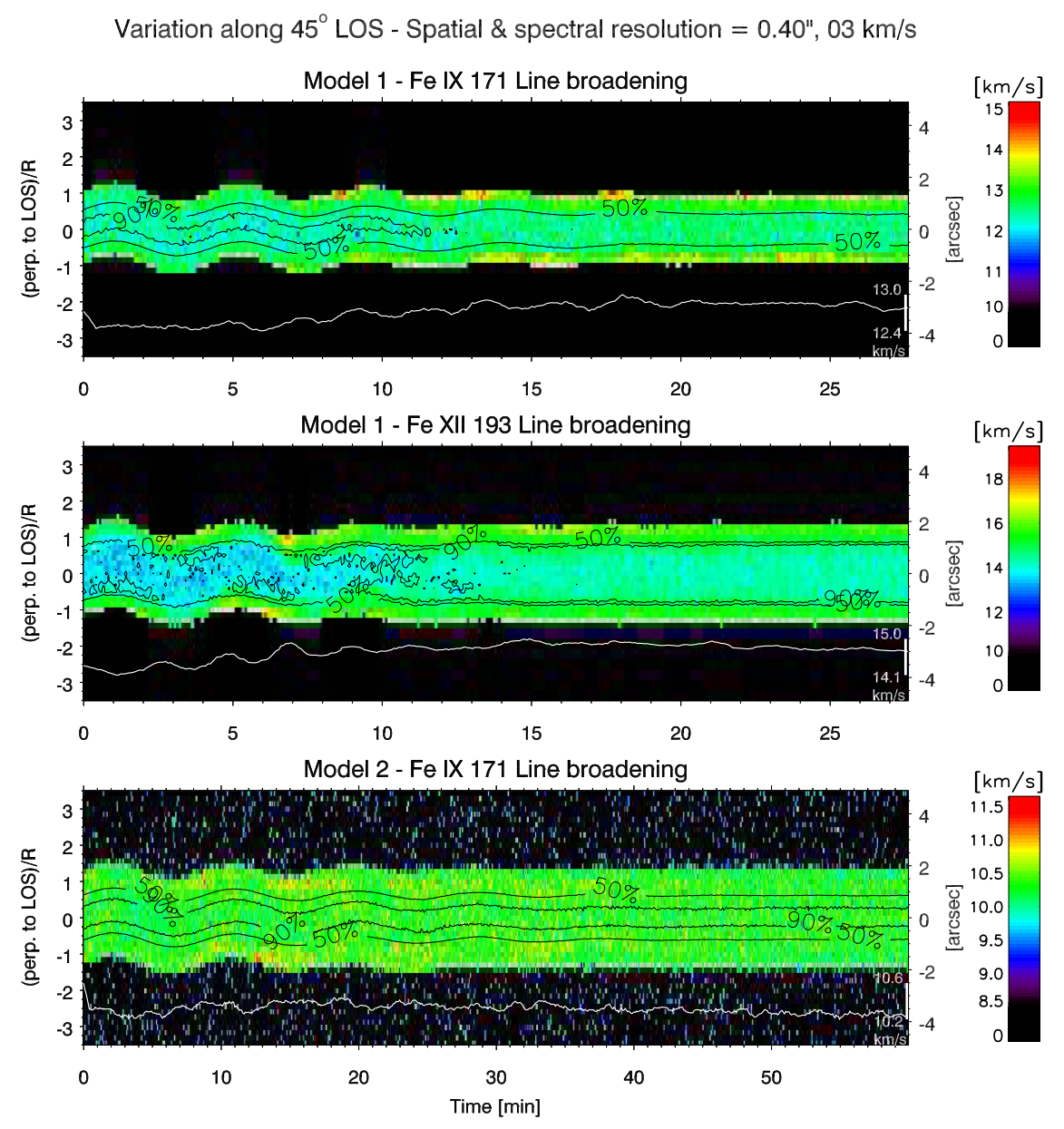

Figure 16. Similar to Figure 13, but for the line width (see also Figure 10 for further explanation). The target instrument has a spatial resolution of 0 "! 4 and a spectral resolution of $3 \mathrm{~km} \mathrm{~s}^{-1}$. The intensity contours with the corresponding spatial resolution are overlaid in each diagram, enclosing the regions with $50 \%$ and $90 \%$ intensity signal with respect to the maximum intensity at $t=0$.

only small-scale, low-amplitude perturbations are obtained. Such motions do not alter the line width by more than a few $\mathrm{km} \mathrm{s}^{-1}$, which may explain why the detection of periodicity in the line widths associated with transverse MHD waves is very scarce in the literature. By the end of the simulation, the contribution to the kinetic energy from the non-thermal component is similar to or larger than the contribution from the Doppler velocity. Importantly, the average decrease in the Doppler velocity by a factor of 10 between the first and last stages of the simulation is not compensated for by a similar increase in the observable non-thermal line widths.

Since the initial kink perturbations end up in localized azimuthal motions due to resonant absorption, with phase mixing and the KHI further reducing the spatial scales and possibly leading to local turbulence-like behavior, our model supports a positive correlation between Doppler velocities and line widths, as found, for instance, in McIntosh \& De Pontieu (2012). However, our results also suggest that positive correlations between Doppler velocities and line widths are limited, since a significant line width increase is not obtained. This is particularly limiting for estimating the true wave energy present in the solar corona. Based on our results, we estimate the hidden energy from the wave to be a factor of about 5-10 of the observed value. Our results also predict an increase in the high-frequency waves and small scales accompanying these processes, perhaps matching the observations by De Moortel et al. (2014). This is supported by numerical results from Magyar \& Van Doorsselaere (2016) for a very similar model. The power spectra of velocity fluctuations may therefore provide an additional tool for constraining the wave energy.

Our model further predicts a positive/negative correlation between line width and intensity for a boundary/core line in a non-uniform temperature model of a loop colder than the outside. The opposite correlation would be found in the case of a hot loop. On the other hand, if the loop presents no significant temperature variation, then a mild negative correlation between intensity and line width should be present (assuming that the $\mathrm{KHI}$ does not contribute significantly to the heating, as is the case in model 2). Anti-correlations that may match this scenario have been found with Hinode/EIS (Scott \& Martens 2011).

When investigating specific events of loops undergoing a single transverse perturbation, as is often the case during flares (but limited to cases of small-amplitude perturbation), our results also predict a negative correlation in time between Doppler velocities and line width, especially when observing at high spatial resolution (enough to resolve the resonant component in the Doppler maps). Although such negative correlations have been observed in active regions with Hinode/ EIS (Doschek et al. 2007), caution must be taken when comparing to such reports since in our work only the transverse velocity components to the loop are considered (the LOS rays are always perpendicular to the axis of the loop). While the 


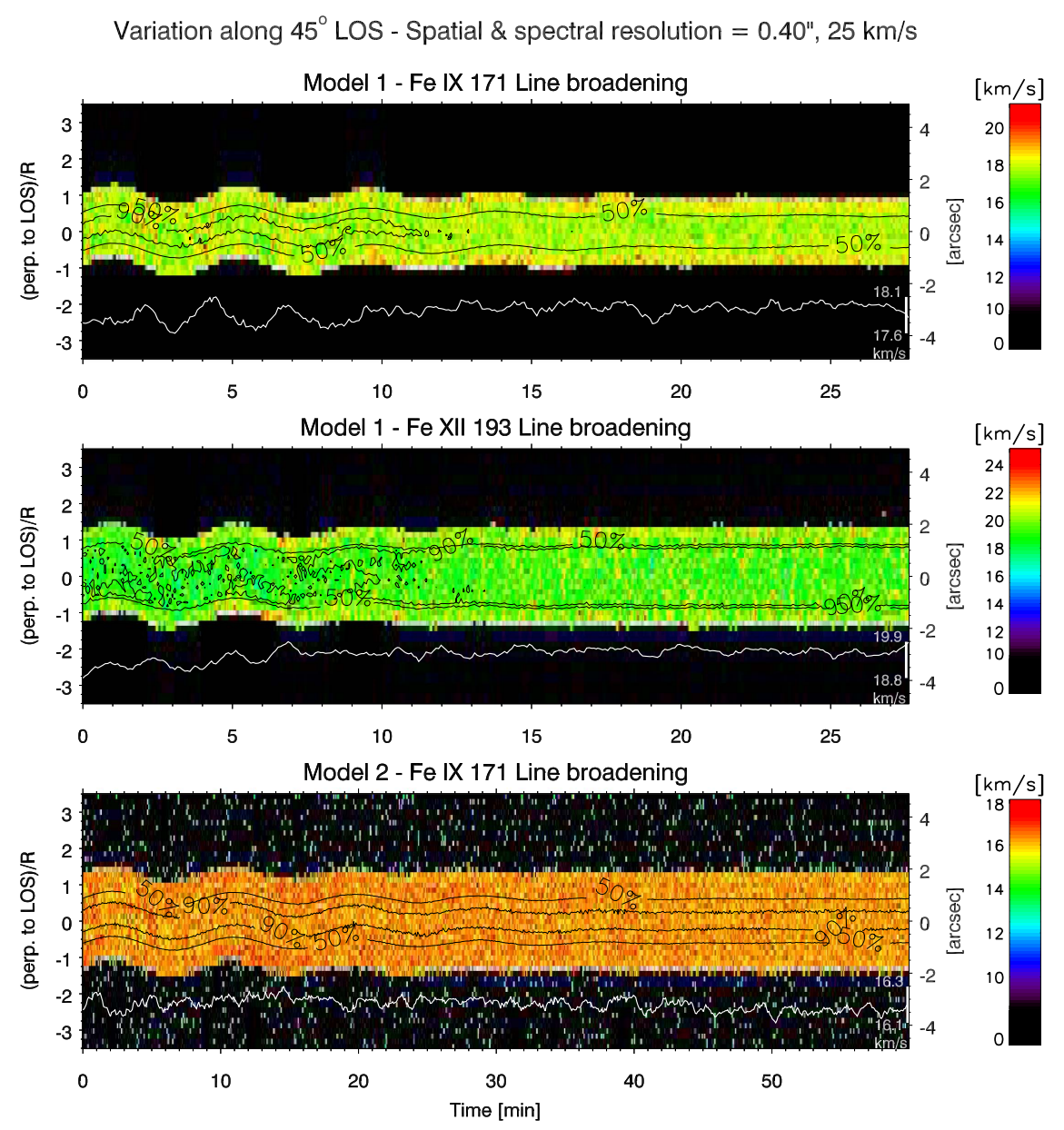

Figure 17. Similar to Figure 16, but with a spectral resolution of $25 \mathrm{~km} \mathrm{~s}^{-1}$.

kink perturbation does produce longitudinal flows in our model (up to a few $\mathrm{km} \mathrm{s}^{-1}$, depending on the amplitude), they are far lower than the values found in active regions, tens to hundreds of $\mathrm{km} \mathrm{s}^{-1}$ (see, e.g., Reale 2010 and references therein).

\section{Conclusions}

We have investigated the observational signatures of transverse MHD waves by performing 3D MHD numerical simulations. We have focused on the case of standing (global) kink waves in coronal flux tubes (with radial density structuring) and considered the signatures for both imaging and spectroscopic devices. We have further taken two different coronal models, with and without a temperature variation in the perpendicular cross-section, and considered two spectral lines, the "core" and "boundary" lines, catching more of the dynamics of the loop core and boundary, respectively. Modulation of intensity, Doppler velocities, line width, and loop width are obtained, mainly due to the combination of the $\mathrm{KHI}$ and resonant absorption, and to a minor degree to the inertia and fluting modes produced by the initial kink mode. It is important to realize therefore that non-linear effects such as those obtained in our model can lead to line width and loop width modulations that would usually be associated with sausage modes (Antolin \& Van Doorsselaere 2013). Long, non-periodic trends include dimming/enhancement and loop width thinning/broadening in the core/boundary line, and preto post-KHI positive jumps of intensity, Doppler, and line widths. In Table 1, we summarize the phase relations and main features of the observable quantities, combined with previous findings (Antolin et al. 2015, 2016). Despite the relatively simple model, we can see that several factors combine to create a very complex set of observable features. This illustrates how hard it must be to disentangle all of the physical mechanisms in real observations. Our main results are the following.

1. Fine strand-like structure increases for spectral lines catching the boundary dynamics.

2. The apparent decay-less oscillations produced by TWIKH rolls (Antolin et al. 2016) are a unique feature of non-uniform temperature models.

3. The intensity power peak is dominated by KHI at half the kink period. The broadening of the peak in the global wavelet transform is mostly due to temperature and increases for uniform cross-section temperature models, thereby serving as a diagnostic tool of the inhomogeneous boundary layer.

4. A small out-of-phase periodic brightening is obtained between the core and boundary line intensities with a period double that of the kink mode. The line widths and loop widths have double periodicity and their phases with respect to the displacement depend on the LOS angle.

5. A characteristic arrow-shaped structure is obtained in Doppler velocity time-distance diagrams, with a ragged Doppler transition at high spatial resolution and a maximum Doppler signal toward the edges of the flux 

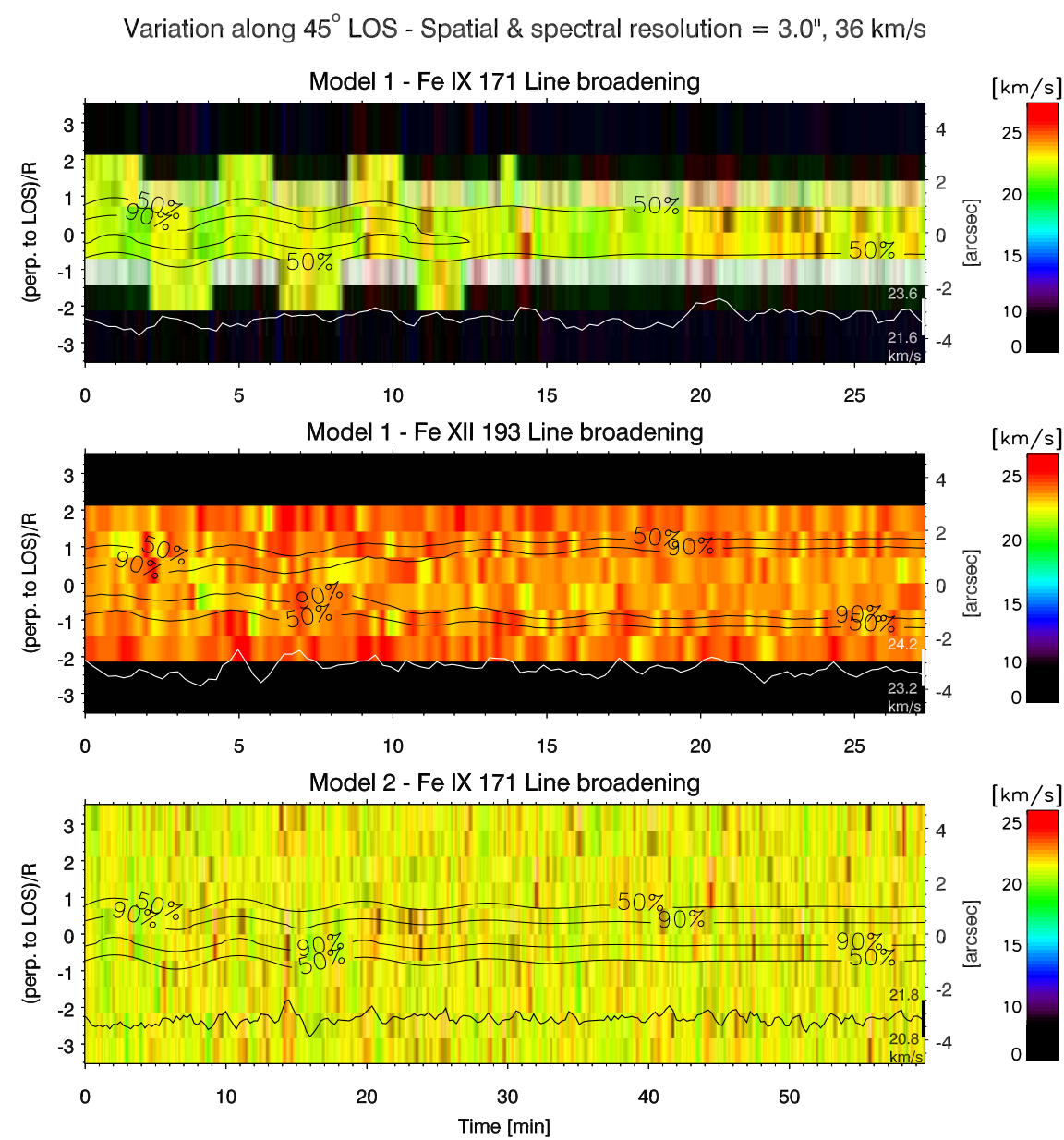

Figure 18. Similar to Figure 16, but with a spatial resolution of $3^{\prime \prime}$, a spectral resolution of $36 \mathrm{~km} \mathrm{~s}^{-1}$, and a temporal resolution of $15 \mathrm{~s}$ targeting Hinode/EIS.

Table 1

Observational Signatures of the Kink Mode with TWIKH Rolls

\begin{tabular}{|c|c|c|c|}
\hline $\begin{array}{l}\text { Spectral } \\
\text { Line }\end{array}$ & Quantity & (Spatial and Temporal) & Large Scale \\
\hline Core & $\begin{array}{l}\text { Morphology } \\
\text { Displacement } \xi_{C} \\
\text { Intensity } I_{C} \\
\text { Dopper velocity } \\
\text { Line width } \\
\text { Loop width }\end{array}$ & $\begin{array}{l}\text { Stranded } \\
\mathrm{P} ; \text { phases out with } \xi_{B} \\
\mathrm{P} / 2(0.1 \%) ; \text { out } / \mathrm{ph}[\pi] \text { with } I_{B} \\
\mathrm{P} ; \text { out } / \mathrm{ph}\left[\frac{\pi}{2}-\pi\right] \text { with } \xi_{C} ; \text { ragged; enhanced boundaries }(50 \%) \\
\mathrm{P} / 2(3 \%) ; \text { out } / \mathrm{ph}[\pi] \text { with } I_{C} ; \text { enhanced boundaries }\left(10 \%^{\mathrm{b}}-20 \%\right) \\
\mathrm{P} / 2\left(4 \%^{\mathrm{b}}-10 \%\right) ; \text { out } / \mathrm{ph} \text { with } I_{C}\end{array}$ & $\begin{array}{l}\text { Monolithic } \\
\text { Gaussian damping, } \\
\searrow\left(3 \% \%^{\mathrm{b}}-20 \%\right) \\
\text { Arrow; \(90\%) } \\
\nearrow\left(2 \%^{\mathrm{b}}-5 \%\right) \\
\searrow\left(3 \%^{\mathrm{b}}-15 \%\right)\end{array}$ \\
\hline
\end{tabular}

Notes. $P$ denotes the period of the kink mode. $P / 2$ denotes double periodicity. Small spatial and temporal scales denote scales on the order of $1 / 3$ of the loop radius and time changes on the order of the kink period or less. The $\nearrow$ and $\searrow$ symbols indicate increasing and decreasing amplitudes in the long term, respectively. The percentage next to the respective variation indicates its average magnitude. "out/ph" and "in/ph" mean out-of-phase and in-phase relations, respectively. The LOSdependent phase values are for a $0^{\circ}$ LOS. All phase relations correspond to that observed after the onset of the KHI.

${ }^{a}$ Phase is LOS dependent.

${ }^{\mathrm{b}}$ Values for the uniform temperature model (model 2). 


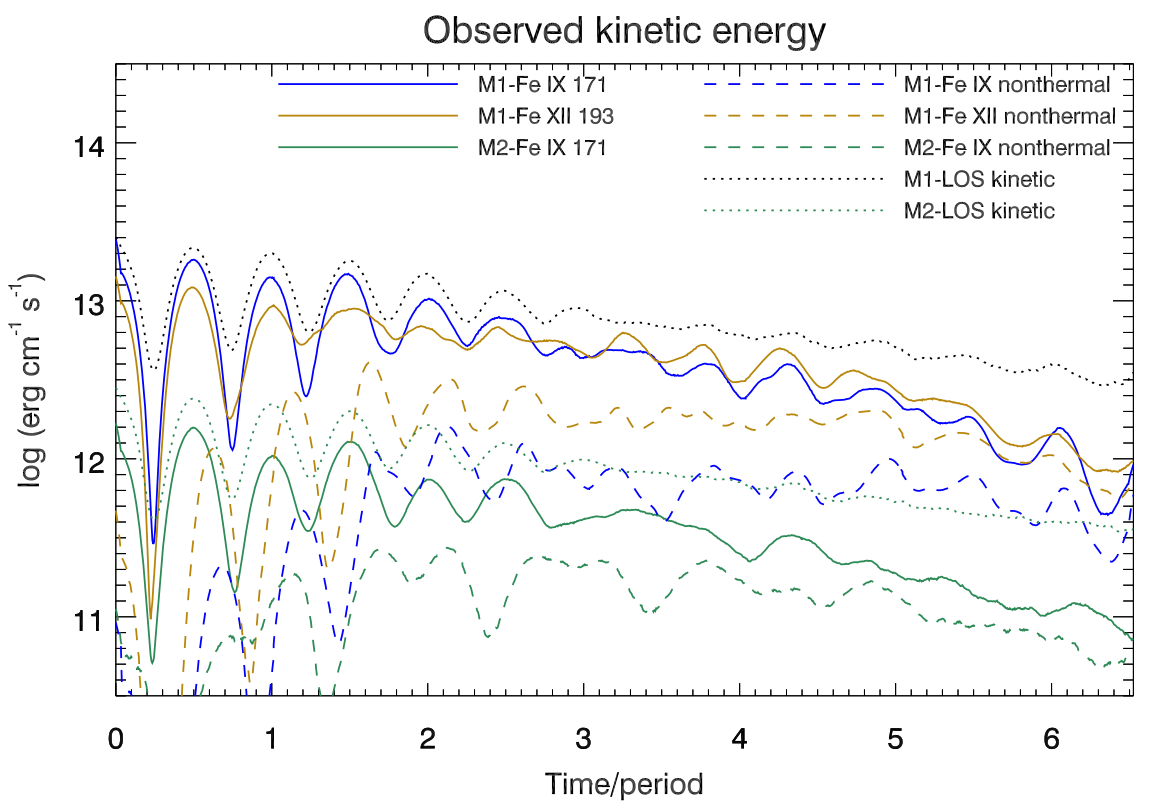

Figure 19. Observed kinetic energy flux at the loop apex for each model (solid curves), given by Equation (3). The true input value at the loop apex, at full numerical resolution and for a $45^{\circ} \mathrm{LOS}$, given by Equation (7), is shown in dotted lines for each model. The dashed curves show the kinetic energy flux provided by the nonthermal component only (which is Equation (3) without the Doppler term). The blue and dark gold colors correspond to the values derived, respectively, from the Fe IX 171 and Fe XII 193 lines in model 1. The green colored curves correspond to the values derived from the Fe IX 171 line in model 2.

tube which can dominate the overall signal. This is accompanied by an overall spectral line broadening of a few $\mathrm{km} \mathrm{s}^{-1}$.

6. Both the displacement and the Doppler velocities of the core and boundary lines become out-of-phase over time, especially at coarse spatial resolution.

7. The above spectral features can be captured for a spectral resolution of $25 \mathrm{~km} \mathrm{~s}^{-1}$ and spatial resolution of 0 !" 33, and most are lost for a coarse spatial (and spectral) resolution such as that of Hinode/EIS. When reducing the spectral resolution from 3 to $25 \mathrm{~km} \mathrm{~s}^{-1}$, the line widths are significantly overestimated by $40 \%-60 \%$. Overall, we find that spectral resolution affects the line width measurements much more than the Doppler velocity measurements.

8. The estimation of the wave energy based on the observed Doppler velocities at the end of the simulation would therefore provide a very inaccurate value of about $10 \%$ the initial wave energy. The non-thermal contribution to the observed kinetic energy is similar to that from the Doppler motions. The average decrease in the Doppler velocity by a factor of 10 between the first and last stage of the simulation is not observationally compensated for by a similar increase in the non-thermal line widths. We estimate this discrepancy to be a factor of about 5-10 of the observed value.

This research has received funding from the UK Science and Technology Facilities Council and the European Union Horizon 2020 research and innovation programme (grant agreement No. 647214), and also from JSPS KAKENHI grant No. 25220703 (PI: S. Tsuneta) and 15H03640 (PI: T. Yokoyama). T.V.D. was supported by FWO Vlaanderen's Odysseus programme, GOA-2015-014 (KU Leuven) and the IAP P7/08 CHARM (Belspo). Numerical computations were carried out on Cray XC30 at the Center for Computational
Astrophysics, NAOJ. This work also benefited from the ISSICoronal Rain (P.I. Patrick Antolin) and ISSI-BJ meetings (P.I. V. Nakariakov \& T. Van Doorsselaere).

\section{References}

Allan, W., \& Wright, A. N. 2000, JGR, 105, 317

Anfinogentov, S., Nisticò, G., \& Nakariakov, V. M. 2013, A\&A, 560, A107

Anfinogentov, S. A., Nakariakov, V. M., \& Nisticò, G. 2015, A\&A, 583, A136 Antolin, P., De Moortel, I., Van Doorsselaere, T., \& Yokoyama, T. 2016, ApJL, 830, L22

Antolin, P., Okamoto, T. J., De Pontieu, B., et al. 2015, ApJ, 809, 72

Antolin, P., \& Van Doorsselaere, T. 2013, A\&A, 555, A74

Antolin, P., Yokoyama, T., \& Van Doorsselaere, T. 2014, ApJL, 787, L22

Arregui, I., Oliver, R., \& Ballester, J. L. 2012, LRSP, 9, 2

Aschwanden, M. J., Fletcher, L., Schrijver, C. J., \& Alexander, D. 1999, ApJ, 520,880

Asgari-Targhi, M., van Ballegooijen, A. A., \& Imada, S. 2014, ApJ, 786, 28 Brannon, S. R., Longcope, D. W., \& Qiu, J. 2015, ApJ, 810, 4

Cally, P. S., Moradi, H., \& Rajaguru, S. P. 2016, GMS, 216, 489

Cargill, P. J., De Moortel, I., \& Kiddie, G. 2016, ApJ, 823, 31

Cranmer, S. R., \& Woolsey, L. N. 2015, ApJ, 812, 71

De Moortel, I., McIntosh, S. W., Threlfall, J., Bethge, C., \& Liu, J. 2014, ApJL, 782, L34

De Moortel, I., \& Nakariakov, V. M. 2012, RSPTA, 370, 3193

De Moortel, I., \& Pascoe, D. J. 2012, ApJ, 746, 31

De Moortel, I., Pascoe, D. J., Wright, A. N., \& Hood, A. W. 2016, PPCF, 58, 014001

De Pontieu, B., McIntosh, S., Martinez-Sykora, J., Peter, H., \& Pereira, T. M. D. 2015, ApJL, 799, L12

Dere, K. P., Landi, E., Young, P. R., et al. 2009, A\&A, 498, 915

Doschek, G. A., Mariska, J. T., Warren, H. P., et al. 2007, ApJL, 667, L109

Goossens, M., Andries, J., \& Arregui, I. 2006, RSPTA, 364, 433

Goossens, M., Andries, J., \& Aschwanden, M. J. 2002, A\&A, 394, L39

Goossens, M., Andries, J., Soler, R., et al. 2012, ApJ, 753, 111

Goossens, M., Erdélyi, R., \& Ruderman, M. S. 2011, SSRv, 158, 289

Goossens, M., Soler, R., Terradas, J., Van Doorsselaere, T., \& Verth, G. 2014, ApJ, 788, 9

Gruszecki, M., Nakariakov, V. M., van Doorsselaere, T., \& Arber, T. D. 2010, PhRvL, 105, 055004

Hara, H., \& Ichimoto, K. 1999, ApJ, 513, 969

Hara, H., Watanabe, T., Harra, L. K., et al. 2008, ApJL, 678, L67

Hollweg, J. V. 1987, ApJ, 312, 880

Hollweg, J. V., \& Yang, G. 1988, JGR, 93, 5423 
Ionson, J. A. 1978, ApJ, 226, 650

Karampelas, K., Van Doorsselare, T., \& Antolin, P. 2017, A\&A, submitted

Karpen, J. T., Antiochos, S. K., Dahlburg, R. B., \& Spicer, D. S. 1993, ApJ 403, 769

Khomenko, E., \& Collados, M. 2015, LRSP, 12, 6

Klimchuk, J. A. 2006, SoPh, 234, 41

Kudoh, T., Matsumoto, R., \& Shibata, K. 1999, IJCFD, 8, 56

Lin, Y. 2011, SSRv, 158, 237

Magyar, N., \& Van Doorsselaere, T. 2016, ApJ, 823, 82

McIntosh, S. W., \& De Pontieu, B. 2012, ApJ, 761, 138

McIntosh, S. W., de Pontieu, B., Carlsson, M., et al. 2011, Natur, 475, 477

Morton, R. J., Tomczyk, S., \& Pinto, R. F. 2016, ApJ, 828, 89

Murawski, K., Chmielewski, P., Zaqarashvili, T. V., \& Khomenko, E. 2016, MNRAS, 459, 2566

Nakariakov, V. M., Anfinogentov, S. A., Nisticò, G., \& Lee, D.-H. 2016, A\&A, 591, L5

Nakariakov, V. M., Ofman, L., Deluca, E. E., Roberts, B., \& Davila, J. M. 1999, Sci, 285, 862

Nisticò, G., Nakariakov, V. M., \& Verwichte, E. 2013, A\&A, 552, A57

Ofman, L., Davila, J. M., \& Steinolfson, R. S. 1994, GeoRL, 21, 2259

Okamoto, T. J., Antolin, P., De Pontieu, B., et al. 2015, ApJ, 809, 71

Okamoto, T. J., Liu, W., \& Tsuneta, S. 2016, ApJ, 831, 126

Parenti, S., \& Vial, J.-C. 2007, A\&A, 469, 1109

Parenti, S. 2015, in Astrophysics and Space Science Library, Vol. 415, ed. J.-C. Vial \& O. Engvold (Berlin: Springer), 61

Pascoe, D. J., Wright, A. N., \& De Moortel, I. 2010, ApJ, 711, 990

Pascoe, D. J., Wright, A. N., \& De Moortel, I. 2011, ApJ, 731, 73
Poedts, S., Toth, G., Belien, A. J. C., \& Goedbloed, J. P. 1997, SoPh, 172, 45

Reale, F. 2010, LRSP, 7, 5

Ruderman, M. S., Goossens, M., \& Andries, J. 2010, PhPl, 17, 082108

Sakurai, T., Goossens, M., \& Hollweg, J. V. 1991, SoPh, 133, 227

Scott, J. T., \& Martens, P. C. H. 2011, ApJ, 742, 101

Terradas, J., Andries, J., Goossens, M., et al. 2008a, ApJL, 687, L115

Terradas, J., Arregui, I., Oliver, R., et al. 2008b, ApJ, 679, 1611

Terradas, J., Goossens, M., \& Verth, G. 2010, A\&A, 524, A23

Tomczyk, S., McIntosh, S. W., Keil, S. L., et al. 2007, Sci, 317,1192

Torrence, C., \& Compo, G. P. 1998, BAMS, 79, 61

Uchida, Y., \& Kaburaki, O. 1974, SoPh, 35, 451

Van Doorsselaere, T., Andries, J., Poedts, S., \& Goossens, M. 2004, ApJ, 606, 1223

Van Doorsselaere, T., Antolin, P., Yuan, D., Reznikova, V., \& Magyar, N. 2016, FrASS, 3, 4

Van Doorsselaere, T., Nakariakov, V. M., \& Verwichte, E. 2008a, ApJL, 676, L73

Van Doorsselaere, T., Nakariakov, V. M., Young, P. R., \& Verwichte, E. 2008b, A\&A, 487, L17

Verth, G., Terradas, J., \& Goossens, M. 2010, ApJL, 718, L102

Viall, N. M., \& Klimchuk, J. A. 2012, ApJ, 753, 35

Wentzel, D. G. 1974, SoPh, 39, 129

Woolsey, L. N., \& Cranmer, S. R. 2015, ApJ, 811, 136

Yuan, D., \& Van Doorsselaere, T. 2016, ApJS, 223, 23

Zaqarashvili, T. V., Zhelyazkov, I., \& Ofman, L. 2015, ApJ, 813, 123

Ziegler, U., \& Ulmschneider, P. 1997, A\&A, 327, 854 